EUROPEAN ORGANIZATION FOR NUCLEAR RESEARCH

CERN-EP/98-169

21 October 1998

\title{
Search for composite and exotic fermions at LEP 2
}

DELPHI Collaboration

\begin{abstract}
A search for unstable heavy fermions with the DELPHI detector at LEP is reported. Sequential and non-canonical leptons, as well as excited leptons and quarks, are considered. The data analysed correspond to an integrated luminosity of about $48 \mathrm{pb}^{-1}$ at an $e^{+} e^{-}$centre-of-mass energy of $183 \mathrm{GeV}$ and about $20 \mathrm{pb}^{-1}$ equally shared between the centre-of-mass energies of $172 \mathrm{GeV}$ and $161 \mathrm{GeV}$. The search for pair-produced new leptons establishes $95 \%$ confidence level mass limits in the region between $70 \mathrm{GeV} / c^{2}$ and $90 \mathrm{GeV} / c^{2}$, depending on the channel. The search for singly produced excited leptons and quarks establishes upper limits on the ratio of the coupling of the excited fermion to its mass $\left(\lambda / m_{f^{*}}\right)$ as a function of the mass.
\end{abstract}

(Accepted by E. Phys. J. C) 


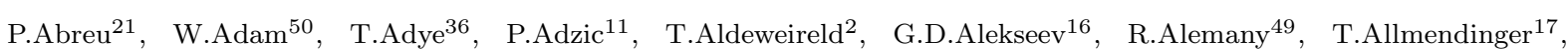
P.P.Allport2 S.Almehed U.Amaldi S.Amato 47, E.G.Anassontzi 3 P.Andersson 44 , A.Andreazza, S.Andringa 21 ,

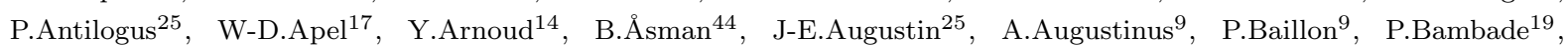
F.Barao21 G.Barbiellini46, R.Barbier 25, D.Y.Bardin16 G.Barkerl, A.Baroncelli38, M.Battaglia15, M.Baubillier 23, K-H.Becks52, M.Begalli6, P.Beilliere8, Yu.Belokopytov 53, A.C.Benvenut 5 , C.Berat14, M.Berggren 25, D.Bertini25, D.Bertrand21, M.Besancon39, F.Bianch 45, M.Bigi45, M.S.Bilenky 16, M-A Bizouard 19, P.Bloch 10 , H.M.Blom 30

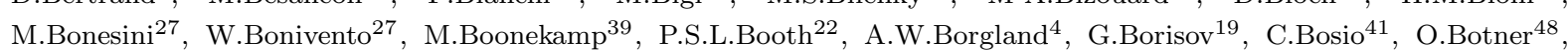

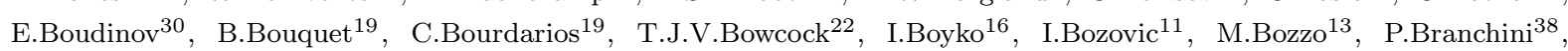
T.Brenke52, R.A.Brennen 48, P.Bruckman 18, J-M.Brunet 8, L.Bugge32, T.Buran 32, T.Burgsmueller 52 , P.Buschmann 52 , S.Cabrera49, M.Caccia27, M.Calv27, A.J.Camacho Rozas 40, T.Camporesi月, V.Canale 37, F.Carena9, L.Carroll22,

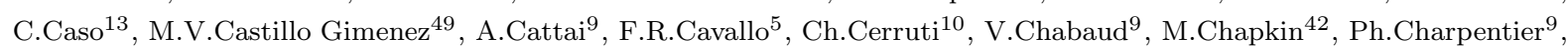

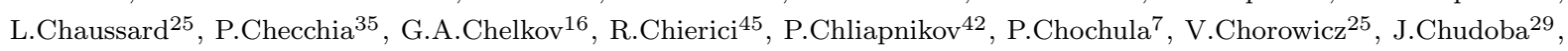

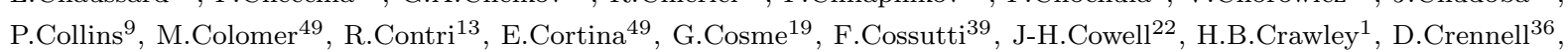

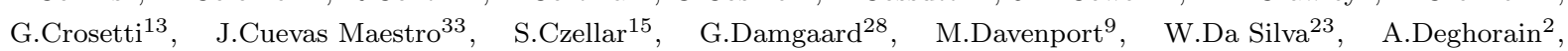

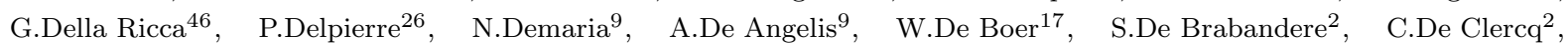
B.De Lotth 16, A.DeMin 35, L.De Paula47, H.Dijkstra9, L.Di_Ciaccio37, ADi Diodato37, J.Dolbeau 8 , K.Doroba51,

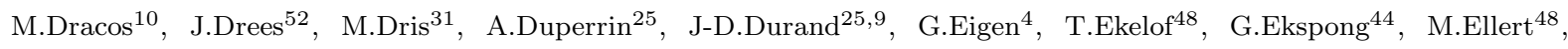
M.Elsing J-P.Enge10 B.Erzen 13 , M.Espirito Santo21, E.Falk24, G.Fanourakis11, D.Fassouliptis11, J.Fayot23, M.Feindt 17, A.Fenyuk12, P.Ferrari27, A.Ferrer 49, E.Ferrer-Ribas 19 , S.Fichet 23, A.Firestonet, P.-A.Fischent, U.Flagmever 52, H.Foeth 9 , E.Fokiti31, F.Fontanell 13, B.Franek 36 A.G.Frodesent, R.Fruhwirth 10 , F.Fulda-Quenzer 19 ,

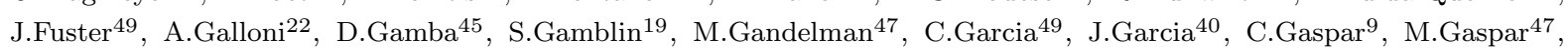
U.Gasparini 35, Ph.Gavillet9, E.N.Gazis3, D.Gele10, J-P.Gerben, L.Gerdyukov42, N.Ghgdbane25, I.Gil149, F.Glege 52 ,

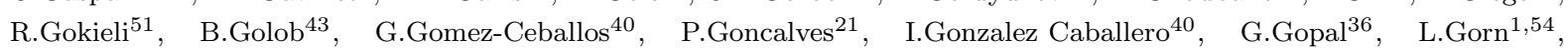

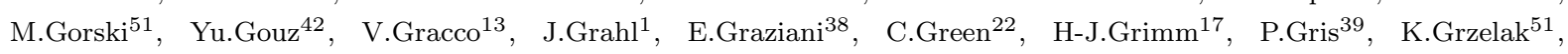

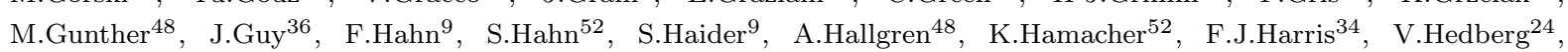
S.Heising 17, J.J.Hernande49, P.Herquet2 H.Herr/, T.L.Hessing 34, J.-M.Heusen 52, E.Higon 49 S-O.Holmgren 44 , P.J.Holt34, D.Holthuizen30, S.Hoorelbeke2, M.Houlden22, J.Hrubed50, KHuet2, K.Hultqvist 44, J.N.Jackson 22,

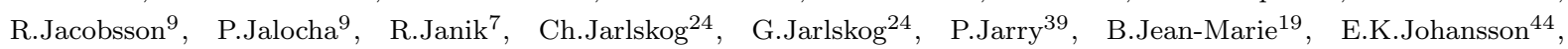
P.Jonsson 24, C.Joram 9, P.Juilloted, F.Kapusta23, K.Karafasoulje11, S.Katsanevas25, E.C_Katsoufis 31, R.Keranen 17,

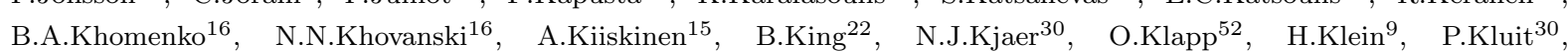

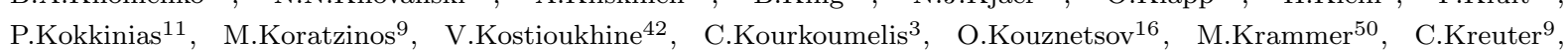

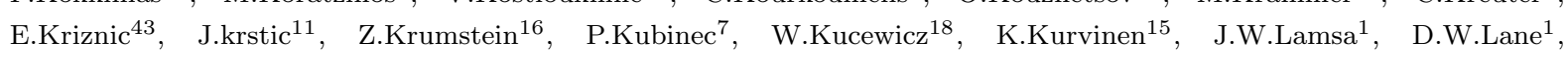
P.Langefeld52, V.Lapin 12 , J-P.Laugier 39 , R.Lauhakangas 15 , G.Leder 50, F.Ledroit 14 , V.Lefebure L.Leinonen 44 , A.Leisos 11, R.Leitner29, G.Lenzen 52 .V.Lepeltier19, T.Lesiak18, M.Lethuillier39, J.Libby 34, D.Likd 9, A.Lipniacka 44, I.Lippi35, B.Loerstad24, J.G.Loken34, JH.Lopes47, J.M.Lope 40, R.Lopez-Fernande214, D.Loukas 11 , P.Lutz 39 , L.Lyon 34, J.MacNaughtonE0, J.R.Mahon6, A.Majo21, A.Malek 25, T.G.M.Malmgren44, V.Malycheve, F.Mand150,

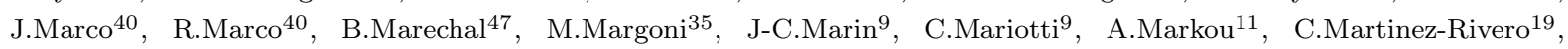
F.Martinez-Vidal196, SMarti i Garcia22, N.Mastroyiannopoulos17, FMatorras 40, _C.Matteuzzi27, G.Matthiae 37, J.Mazik29, F.Mazzucato35, M.Mazzucatd 35, M.Mc Cubbin22, R.Mc Kayt, R.Mc Nulty 9, G.Mc Pherson 22, C.Meroni27,

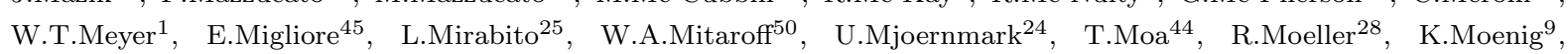

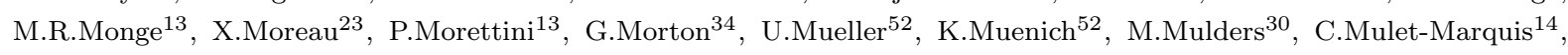
R.Muresan24, W.J.Murray 36, B.Muryn 14 18, G.Mratt34, T.Myklebust32, F.Naraghi14, F.L.Nayarria5, S.Nayas 49,

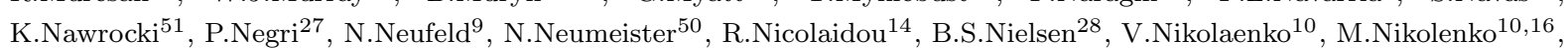
V.Nomokonov15, A.Normand22, A.Nygren 24 V.Obraztsor 42, A.G.Olshevski16, A.Onofre21, R.Oraval 15, G.Orazi10, K.Osterbero 15, A.Quraou39, M.Paganoni27, S.Paianol, R.Pain23, R.Paiva21, J.Palacio 34 H.Palka 18 , Th.D.Papadopoulou 31, K.Papageorgiou 11 L.Pape 9, C.Parkes4, F.Pargd 13, U.Parzefall22, A.Passeri38 O.Passon 52 , M.Pegoraro35, L.Peralta21, M.Pernicka55, A.Perrotta5, C.Petridou46, A.Petrolini13, H.T.Phillips 36 , G.Piana13, F.Pierr 39, M.Piment221 E.Piotto27, T.Podobnik43, M.E.Pold, G.Polok 18, P.Poropat 46,Y.Pozdniakov16, P.Privitera 37 , N.Pukhaeva 16, A.Pullia 27, D.Radojicic34, S.Ragazzi27, H.Rahmani31, D.Rakeczy 56, J.Rames2, P.N.Ratofl20,

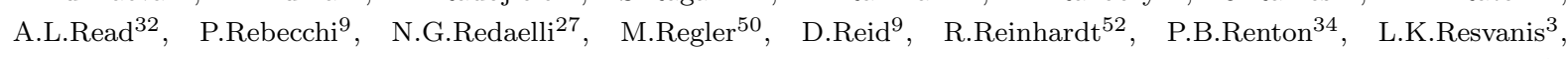

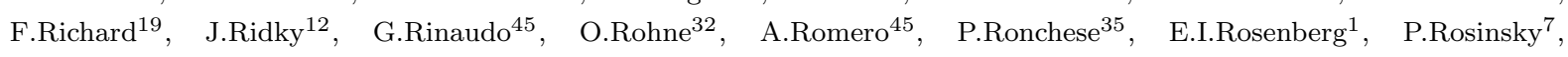

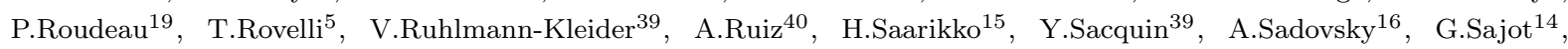

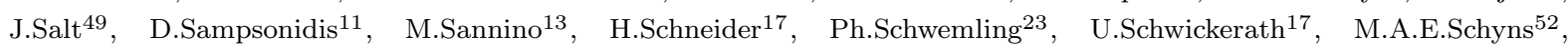
F.Scuri46, P.Seagere0, Y.Sedykh16, A.M.Segan 34, R.Sekulin 36 , R.C.Shellard6, A.Sheridan22, M.Siebe152, R.Silvestre 39 , L.Simard 39, F.Simonetto 35 A.N.Sisakian 16, T.B.Skaali32, G.Smadja25, N.Smirnov42 O.Smirnova24, G.R.Smith 36 , A.Sopczak17, R.Sosnowski51, T.Spassove1, E.Spiriti38, P.Sponhol252, S.Squarciat3, C.Stanescu38, S.Stanid 43 , S.Stapnes 32 , K.Stevenson 34, A.Stocchi19, J.Strauss 50, R.Strub 10, B.Stugul, M.Szczekowsk 51, M.Szeptycka 51, T.Tabarelli27, F.Tegenfeldt 18 , F.Terranova 27, J.Thomas4, A.Tilquin 26, J.Timmermans 30, L.G.Tkatchev 16 , T.Todorov 19 , 


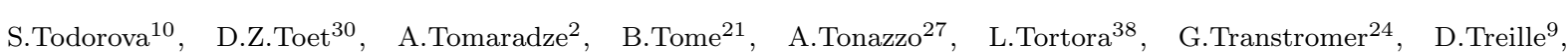

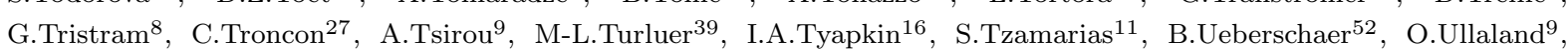
V.Uvarov42, G.Valent5, E.Vallazza 46, G.W.Van Apeldoorn30, P.Van Dam30, J.Van Eldik30 A.Van Lysebettep2 I.Van Vulpen30, N.Vassilopoulo34 G.Vegni27,L.Ventura35, W.Venus36, F.Verbeure2, M.Verlato35, L.S.Vertogradov16,

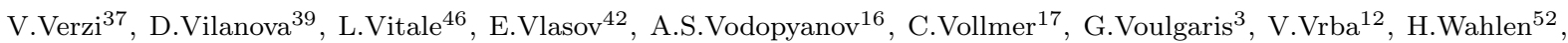

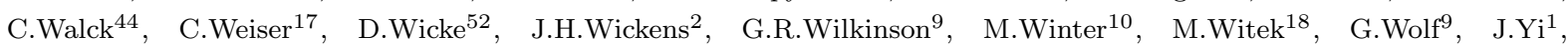
O.Yushchenkd42, A.Zaitsen42, A.Zalewska18, P.Zalewski51, D.Zavrtanik43, E.Zevgolatakos 11, N.I.Zimin 16.24, G.C.Zucchelli 14 , G.Zumerl 35

\footnotetext{
${ }^{1}$ Department of Physics and Astronomy, Iowa State University, Ames IA 50011-3160, USA

${ }^{2}$ Physics Department, Univ. Instelling Antwerpen, Universiteitsplein 1, BE-2610 Wilrijk, Belgium and IIHE, ULB-VUB, Pleinlaan 2, BE-1050 Brussels, Belgium

and Faculté des Sciences, Univ. de l'Etat Mons, Av. Maistriau 19, BE-7000 Mons, Belgium

${ }^{3}$ Physics Laboratory, University of Athens, Solonos Str. 104, GR-10680 Athens, Greece

${ }^{4}$ Department of Physics, University of Bergen, Allégaten 55, NO-5007 Bergen, Norway

${ }^{5}$ Dipartimento di Fisica, Università di Bologna and INFN, Via Irnerio 46, IT-40126 Bologna, Italy

${ }^{6}$ Centro Brasileiro de Pesquisas Físicas, rua Xavier Sigaud 150, BR-22290 Rio de Janeiro, Brazil and Depto. de Física, Pont. Univ. Católica, C.P. 38071 BR-22453 Rio de Janeiro, Brazil

and Inst. de Física, Univ. Estadual do Rio de Janeiro, rua São Francisco Xavier 524, Rio de Janeiro, Brazil

${ }^{7}$ Comenius University, Faculty of Mathematics and Physics, Mlynska Dolina, SK-84215 Bratislava, Slovakia

${ }^{8}$ Collège de France, Lab. de Physique Corpusculaire, IN2P3-CNRS, FR-75231 Paris Cedex 05, France

${ }^{9}$ CERN, CH-1211 Geneva 23, Switzerland

${ }^{10}$ Institut de Recherches Subatomiques, IN2P3 - CNRS/ULP - BP20, FR-67037 Strasbourg Cedex, France

${ }^{11}$ Institute of Nuclear Physics, N.C.S.R. Demokritos, P.O. Box 60228, GR-15310 Athens, Greece

${ }^{12}$ FZU, Inst. of Phys. of the C.A.S. High Energy Physics Division, Na Slovance 2, CZ-180 40, Praha 8, Czech Republic

${ }^{13}$ Dipartimento di Fisica, Università di Genova and INFN, Via Dodecaneso 33, IT-16146 Genova, Italy

${ }^{14}$ Institut des Sciences Nucléaires, IN2P3-CNRS, Université de Grenoble 1, FR-38026 Grenoble Cedex, France

${ }^{15}$ Helsinki Institute of Physics, HIP, P.O. Box 9, FI-00014 Helsinki, Finland

${ }^{16}$ Joint Institute for Nuclear Research, Dubna, Head Post Office, P.O. Box 79, RU-101 000 Moscow, Russian Federation

${ }^{17}$ Institut für Experimentelle Kernphysik, Universität Karlsruhe, Postfach 6980, DE-76128 Karlsruhe, Germany

${ }^{18}$ Institute of Nuclear Physics and University of Mining and Metalurgy, Ul. Kawiory 26a, PL-30055 Krakow, Poland

${ }^{19}$ Université de Paris-Sud, Lab. de l'Accélérateur Linéaire, IN2P3-CNRS, Bât. 200, FR-91405 Orsay Cedex, France

${ }^{20}$ School of Physics and Chemistry, University of Lancaster, Lancaster LA1 4YB, UK

${ }^{21}$ LIP, IST, FCUL - Av. Elias Garcia, 14-1 ${ }^{\circ}$, PT-1000 Lisboa Codex, Portugal

${ }^{22}$ Department of Physics, University of Liverpool, P.O. Box 147, Liverpool L69 3BX, UK

${ }^{23}$ LPNHE, IN2P3-CNRS, Univ. Paris VI et VII, Tour 33 (RdC), 4 place Jussieu, FR-75252 Paris Cedex 05, France

${ }^{24}$ Department of Physics, University of Lund, Sölvegatan 14, SE-223 63 Lund, Sweden

${ }^{25}$ Université Claude Bernard de Lyon, IPNL, IN2P3-CNRS, FR-69622 Villeurbanne Cedex, France

${ }^{26}$ Univ. d'Aix - Marseille II - CPP, IN2P3-CNRS, FR-13288 Marseille Cedex 09, France

${ }^{27}$ Dipartimento di Fisica, Università di Milano and INFN, Via Celoria 16, IT-20133 Milan, Italy

${ }^{28}$ Niels Bohr Institute, Blegdamsvej 17, DK-2100 Copenhagen $\varnothing$, Denmark

${ }^{29} \mathrm{NC}$, Nuclear Centre of MFF, Charles University, Areal MFF, V Holesovickach 2, CZ-180 00, Praha 8, Czech Republic

${ }^{30}$ NIKHEF, Postbus 41882, NL-1009 DB Amsterdam, The Netherlands

${ }^{31}$ National Technical University, Physics Department, Zografou Campus, GR-15773 Athens, Greece

${ }^{32}$ Physics Department, University of Oslo, Blindern, NO-1000 Oslo 3, Norway

${ }^{33}$ Dpto. Fisica, Univ. Oviedo, Avda. Calvo Sotelo s/n, ES-33007 Oviedo, Spain

${ }^{34}$ Department of Physics, University of Oxford, Keble Road, Oxford OX1 3RH, UK

${ }^{35}$ Dipartimento di Fisica, Università di Padova and INFN, Via Marzolo 8, IT-35131 Padua, Italy

${ }^{36}$ Rutherford Appleton Laboratory, Chilton, Didcot OX11 OQX, UK

${ }^{37}$ Dipartimento di Fisica, Università di Roma II and INFN, Tor Vergata, IT-00173 Rome, Italy

${ }^{38}$ Dipartimento di Fisica, Università di Roma III and INFN, Via della Vasca Navale 84, IT-00146 Rome, Italy

${ }^{39}$ DAPNIA/Service de Physique des Particules, CEA-Saclay, FR-91191 Gif-sur-Yvette Cedex, France

${ }^{40}$ Instituto de Fisica de Cantabria (CSIC-UC), Avda. los Castros s/n, ES-39006 Santander, Spain

${ }^{41}$ Dipartimento di Fisica, Università degli Studi di Roma La Sapienza, Piazzale Aldo Moro 2, IT-00185 Rome, Italy

${ }^{42}$ Inst. for High Energy Physics, Serpukov P.O. Box 35, Protvino, (Moscow Region), Russian Federation

${ }^{43}$ J. Stefan Institute, Jamova 39, SI-1000 Ljubljana, Slovenia and Department of Astroparticle Physics, School of Environmental Sciences, Kostanjeviska 16a, Nova Gorica, SI-5000 Slovenia, and Department of Physics, University of Ljubljana, SI-1000 Ljubljana, Slovenia

${ }^{44}$ Fysikum, Stockholm University, Box 6730, SE-113 85 Stockholm, Sweden

${ }^{45}$ Dipartimento di Fisica Sperimentale, Università di Torino and INFN, Via P. Giuria 1, IT-10125 Turin, Italy

${ }^{46}$ Dipartimento di Fisica, Università di Trieste and INFN, Via A. Valerio 2, IT-34127 Trieste, Italy and Istituto di Fisica, Università di Udine, IT-33100 Udine, Italy

${ }^{47}$ Univ. Federal do Rio de Janeiro, C.P. 68528 Cidade Univ., Ilha do Fundão BR-21945-970 Rio de Janeiro, Brazil

${ }^{48}$ Department of Radiation Sciences, University of Uppsala, P.O. Box 535, SE-751 21 Uppsala, Sweden

${ }^{49}$ IFIC, Valencia-CSIC, and D.F.A.M.N., U. de Valencia, Avda. Dr. Moliner 50, ES-46100 Burjassot (Valencia), Spain

${ }^{50}$ Institut für Hochenergiephysik, Österr. Akad. d. Wissensch., Nikolsdorfergasse 18, AT-1050 Vienna, Austria

${ }^{51}$ Inst. Nuclear Studies and University of Warsaw, Ul. Hoza 69, PL-00681 Warsaw, Poland

${ }^{52}$ Fachbereich Physik, University of Wuppertal, Postfach 100 127, DE-42097 Wuppertal, Germany

${ }^{53}$ On leave of absence from IHEP Serpukhov

${ }^{54}$ Now at University of Florida
} 


\section{Introduction}

It is widely believed that the Standard Model (SM), although extremely successful at the present energy scale, is not the final theory. Many possible extensions of the SM discussed in the literature [1] predict the existence of new fermions.

This paper reports a search for unstable exotic and excited leptons and for excited quarks in DELPHI at centre-of-mass energies, $\sqrt{s}$, of $183 \mathrm{GeV}, 172 \mathrm{GeV}$ and $161 \mathrm{GeV}$. Partial results published by DELPHI at $\sqrt{s}=161 \mathrm{GeV}$ can be found in [2]. The statistics correspond to an integrated luminosity of $47.7 \mathrm{pb}^{-1}$ at $\sqrt{s}=183 \mathrm{GeV}, 10 \mathrm{pb}^{-1}$ at $\sqrt{s}=172 \mathrm{GeV}$ and $10 \mathrm{pb}^{-1}$ at $\sqrt{s}=161 \mathrm{GeV}$.

The exotic leptons examined here belong to two classes: sequential and non-canonical. Sequential leptons have gauge quantum numbers identical to the SM leptons (as for instance the hypothetical heavy fourth-generation leptons [3]) while non-canonical leptons [4] have Left-Handed (LH) and Right-Handed (RH) components transforming differently from those of SM leptons?. Two types of non-canonical leptons are searched for: mirror leptons which have the opposite chiral properties of SM leptons, and vector leptons which have both LH and RH components as isodoublets. The production and decay modes of sequential and non-canonical leptons are discussed in sections 2.1 and 2.2 below.

Excited fermions $\left(f^{*}\right)$ are expected in models with substructure in the fermionic sector. Following the simplest phenomenological models [6], excited fermions are assumed to have both spin and isospin 1/2 and to have both their LH and RH components in weak isodoublets (vector-like). Form factors and anomalous magnetic moments of excited leptons are not considered in the present analysis. The production and decay modes of excited leptons and quarks are discussed in section 2.3.

Previous limits set by DELPHI and by other experiments can be found in references [2,7, 10] and [8] respectively.

This paper is organized as follows. In section 2 the production and decay mechanisms of excited and exotic fermions (within the considered models) are discussed. In section 3 the DELPHI detector and the used data samples are briefly described. The event selection and topological classification are discussed in section 4, and the results are presented in sections 5 and 6 .

\section{Production and decay of unstable new fermions}

The new fermions considered in this paper couple to the photon and/or to the $W / Z$ gauge bosons, according to their internal quantum numbers and thus could be pairproduced at LEP. Single production in association with their SM partners is also possible but its rate depends on the $f f^{*} V$ couplings, where $V$ is a generic gauge boson $(V=\gamma, W, Z)$ [ [1]. Excited fermion masses up to $\sqrt{s}$ can be probed through single production, depending on the scale $\Lambda$ of the substructure (which determines the coupling). In the case of excited quarks only the single production modes will be considered. For exotic leptons single production occurs because of their mixing with SM leptons. The mixing angles, severely restricted by data taken at LEP1 and in several low-energy experiments (in particular by the experimental absence of flavour-changing neutral currents), are constrained to be smaller than $\mathcal{O}\left(10^{-1}\right)$ [9]. Given these limits and the present luminosities, exotic single lepton production is not relevant in most scenarios and will not be considered in the present paper.

\footnotetext{
${ }^{1}$ The designation exotic leptons is, for some authors, equivalent to non-canonical leptons, while for others, as in this paper, it encompasses both sequential and non-canonical leptons.
} 
In this paper new fermions are assumed to decay promptly (decay length shorter than about $1 \mathrm{~cm})$. This constraint implies mixing angles greater than $\mathcal{O}\left(10^{-5}\right)$ for exotic leptons. The mean lifetime of excited fermions with masses above $20 \mathrm{GeV} / c^{2}$ is predicted to be less than $10^{-15}$ seconds in all the cases studied.

\subsection{Sequential leptons}

In $e^{+} e^{-}$collisions the pair production of heavy sequential leptons could proceed through $s$-channel $\gamma$ and $Z$ exchange for charged leptons $\left(L^{+} L^{-}\right)$, while for neutral leptons $\left(L^{0} \overline{L^{0}}\right)$ the $\gamma$ channel is absent. There is a $t$-channel $\mathrm{W}$ exchange diagram for $L^{0} \overline{L^{0}}$ which can be neglected, since this contribution involves the suppressed mixing with the first generation.

The cross-sections, given in reference [3], are essentially the SM cross-section for the second and third generations reduced by phase-space factors that are functions of the heavy lepton mass and of the lepton type.

Charged heavy leptons would decay through mixing into one of the lighter neutrinos or charged leptons and a $W^{*}$ or a $Z^{*}$ (for heavy lepton masses above $m_{W}$ and $m_{Z}$, the $W$ or $Z$ will be on-shell): $L^{-} \rightarrow \nu_{\ell} W^{*-}$ or $L^{-} \rightarrow \ell^{-} Z^{*}$, If where $\ell=e, \mu, \tau$.

In a similar way, neutral heavy leptons would be allowed to decay through mixing into a SM charged lepton or neutrino and a $W^{*}$ or a $Z^{*}: L^{0} \rightarrow \ell^{-} W^{*+}$ or $L^{0} \rightarrow \nu_{\ell} Z^{*}$, where again $\ell=e, \mu, \tau$.

The decays into a $W$ boson are largely dominant at the presently accessible masses $\left(m \sim m_{W}<m_{Z}\right)$ and are the only ones taken into account.

Cascade decays involving $L^{-}$and $L^{0}$ were not considered, as in any circumstances the lower mass heavy lepton should instead be detected in the corresponding direct production reaction.

Sequential neutrinos are assumed to be Dirac neutrinos in this analysis.

\subsection{Non-canonical leptons}

The non-canonical leptons considered in this paper (mirror and vector leptons) have the same electrical charge as, but different weak isospin from the corresponding SM leptons. Their pair production in $e^{+} e^{-}$collisions is thus similar to that of the sequential leptons discussed above but with different vector and axial couplings to the Z. Crosssections are given in reference [4].

These new leptons mix with the SM leptons but the non-diagonal terms are negligible [9]. They would decay into massive gauge bosons plus their ordinary light partner. The decay modes of charged mirror and vector leptons $\left(E_{\ell}^{ \pm}\right)$are: $E_{\ell}^{-} \rightarrow \nu_{\ell} W^{*-} ; E_{\ell}^{-} \rightarrow$ $\ell^{-} Z^{* 0}$. The decay modes of neutral mirror and vector leptons $\left(N_{\ell}\right)$ are: $N_{\ell} \rightarrow \ell^{-} W^{*+}$; $N_{\ell} \rightarrow \nu_{\ell} Z^{* 0}$.

The decays into a $Z$ boson have a low BR at the presently accessible masses $\left(m \sim m_{W}\right)$ and will not be considered.

As for sequential leptons, cascade decays involving $E^{-}$and $N$ are also not taken into account.

\footnotetext{
${ }^{2}$ In all cases the corresponding decays of the antiparticles are also implied.
} 


\subsection{Excited fermions}

Pair production of charged excited fermions could proceed via $s$-channel $\gamma$ and $Z$ exchanges in $e^{+} e^{-}$collisions, while for excited neutrinos only $Z$ exchange contributes. Although $t$-channel contributions are also possible, they correspond to double de-excitation, and give a negligible contribution to the overall production cross-section [6].

In the single production mode, excited fermions could result from the $s$-channel $\gamma$ and $Z$ exchange. Important additional contributions from t-channel $\gamma$ and $Z$ exchange arise for excited electron production, while t-channel $W$ exchange can be important for the excited electronic neutrino [6]. For the $t$-channel production process, the unexcited beam particle is emitted preferentially at low polar angle and often goes undetected in the beam pipe.

The effective electroweak Lagrangian [6] associated with magnetic transitions from excited fermions $\mathbf{f}^{*}$ to ordinary fermions $\mathbf{f}$ has the form

$$
L_{\mathbf{f f}^{*}}=\frac{1}{2 \Lambda} \overline{\mathbf{f}^{*}} \sigma^{\mu \nu}\left[g f \frac{\tau}{2} \mathbf{W}_{\mu \nu}+g^{\prime} f^{\prime} \frac{Y}{2} B_{\mu \nu}+g_{s} f_{s} \frac{\lambda}{2} \mathbf{G}_{\mu \nu}\right] \mathbf{f}_{L}+\text { h.c. }
$$

where $\Lambda$ corresponds to the compositeness mass scale, the subscript L stands for lefthanded, $g, g^{\prime}$ and $g_{s}$ are the SM gauge coupling constants and the factors $f, f^{\prime}$ and $f_{s}$ are weight factors associated with the three gauge groups $(\mathrm{SU}(2) \times \mathrm{U}(1) \times \mathrm{SU}(3))$. The meaning of these couplings and a more extensive discussion of the effective Lagrangian can be found in [6]. With the assumption $|f|=\left|f^{\prime}\right|=\left|f_{s}\right|$, or assuming that only one of the constants $f$ is non-negligible, the cross-section depends simply on the parameter $f / \Lambda$, which is related to the excited fermion mass according to $f / \Lambda=\sqrt{2} \lambda / m_{f^{*}}$, where $\lambda$ is the coupling of the excited fermion.

Excited fermions can decay by radiating a $\gamma, Z$ or $W$. For excited quarks, the gluon radiation transition is also possible, becoming in general the most important decay mode. The decay branching ratios are functions of the $f, f^{\prime}$ and $f_{s}$ coupling parameters of the model. Table 1 in shows the excited leptons' decay branching ratios for some relevant values of $f$ and $f^{\prime}$, and for chosen excited lepton masses.

\begin{tabular}{|l||r|r||r|r|}
\hline \multirow{2}{*}{$\begin{array}{l}\text { Decay } \\
\text { Channel }\end{array}$} & \multicolumn{2}{c||}{$\mathrm{M}=80 \mathrm{GeV} / c^{2}$} & \multicolumn{2}{|c|}{$\mathrm{M}=170 \mathrm{GeV} / c^{2}$} \\
\cline { 2 - 5 } & $f=f^{\prime}$ & $f=-f^{\prime}$ & $f=f^{\prime}$ & $f=-f^{\prime}$ \\
\hline \hline$\ell^{*} \rightarrow \ell \gamma$ & 100 & 0 & 37 & 0 \\
\hline$\ell^{*} \rightarrow \ell Z$ & 0 & 0 & 9 & 36 \\
\hline$\ell^{*} \rightarrow \nu W$ & 0 & 100 & 54 & 64 \\
\hline \hline$\nu^{*} \rightarrow \nu \gamma$ & 0 & 100 & 0 & 37 \\
\hline$\nu^{*} \rightarrow \nu Z$ & 0 & 0 & 36 & 9 \\
\hline$\nu^{*} \rightarrow \ell W$ & 100 & 0 & 64 & 54 \\
\hline
\end{tabular}

Table 1: Predicted branching ratios in \% for excited lepton decays (upper part for excited charged leptons, lower part for excited neutrinos).

For charged excited leptons, the electromagnetic radiative decay is forbidden if $f=-f^{\prime}$, and the decay then proceeds through the $Z$ and $W$ bosons. However, if $f=+f^{\prime}$, the electromagnetic radiative decay branching ratio is close to $100 \%$ for $m_{\ell^{*}}$ below $m_{W}$. It decreases above the $W$ threshold, reaching a value of $37 \%$ for $m_{\ell^{*}}=170 \mathrm{GeV} / c^{2}$. 
For excited neutrinos, the situation is reversed, so that the electromagnetic partial decay width is zero if $f=+f^{\prime}$. However, there is a significant contribution to the total decay width from the electromagnetic radiative decay if $f \neq f^{\prime}$, even if the difference $f-f^{\prime}$ is much smaller than $f$ itself.

In the case of excited quarks, the gluon radiation decay mode in general accounts for more than $80 \%$ of the visible width.

The process $e^{+} e^{-} \rightarrow \gamma \gamma(\gamma)$ can be used to probe compositeness at LEP and thus complement the excited electron direct searches for the mass region above the kinematical threshold. In fact, the contribution of the diagram mediated by a virtual excited electron to the $\gamma \gamma$ production cross section would lead to a modification of the angular distribution. This effect depends on the excited electron mass $m_{e^{*}}$ and on the $e e^{*} \gamma$ coupling, $\lambda$.

\subsection{Final state topologies}

Many topologies could result from the decay of unstable heavy fermions. The different possible production and decay modes are schematically shown in table 2. The possible final states involve isolated leptons, isolated photons, jets, missing energy and missing momentum.

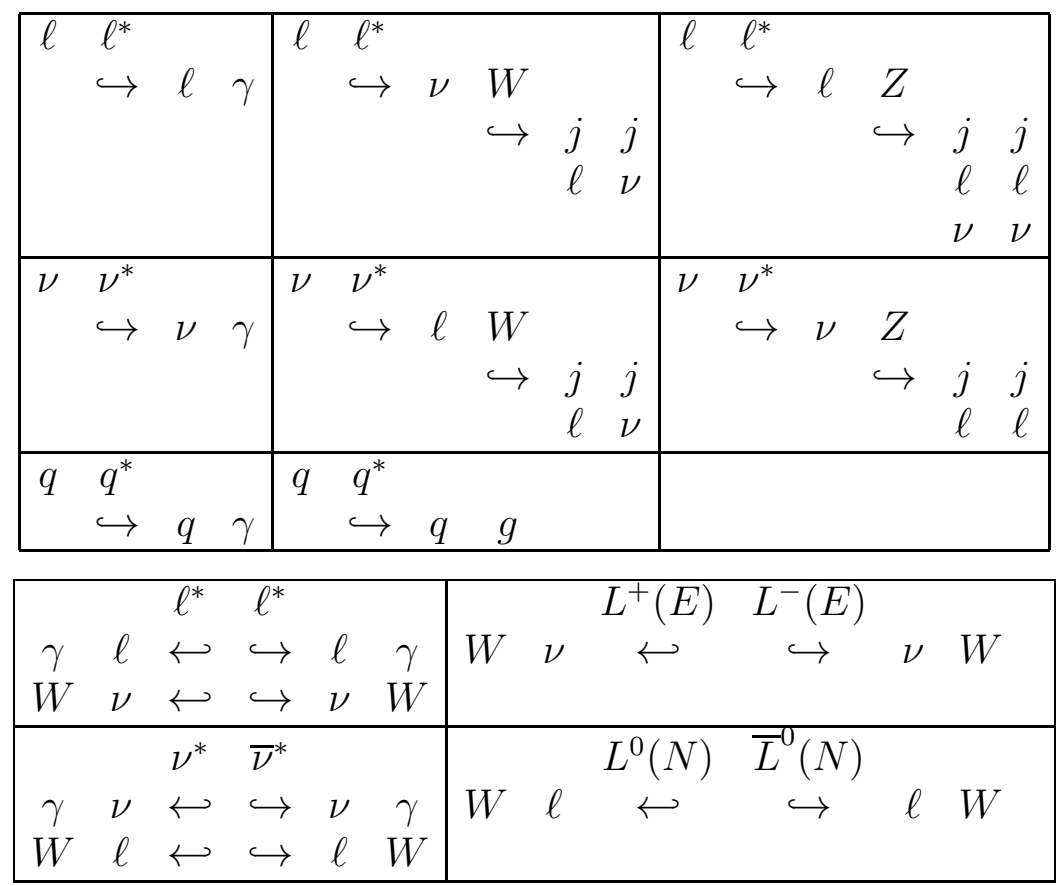

Table 2: Production and decay modes of heavy fermions considered in this analysis. The upper diagrams correspond to single production of excited leptons $\left(\ell^{*}, \nu^{*}\right)$ and quarks $\left(q^{*}\right)$, and the lower diagrams to pair production of excited leptons $\left(\ell^{*}, \nu^{*}\right)$, sequential leptons $\left(L^{ \pm}, L^{0}\right)$, and non-canonical leptons $\left(E_{i}, N_{i}\right)$. The decay products are charged and neutral leptons $(\ell, \nu)$, photons $(\gamma)$, jets $(j)$ and gauge bosons $(\gamma, W, Z, g)$.

In this analysis, the topologies are classified as leptonic if they result from radiative decays of the heavy leptons or from decays into $\mathrm{W}$ or $\mathrm{Z}$ bosons that decay exclusively into leptons, and are classified as hadronic otherwise.

Events can be characterized by the number of jets and the number of isolated leptons and photons as defined by the reconstruction. The different topologies will be referred to as xijk according to the following rule: $x$ is $h$ or $\ell$ for hadronic or leptonic topologies 
and $i$ is the number of jets, $j$ is the number of isolated leptons and $k$ is the number of isolated photons. As an example, h210 is a hadronic topology with two jets and one isolated lepton.

\begin{tabular}{|l|c|c|}
\hline \multirow{2}{*}{ Channel } & \multicolumn{2}{|c|}{ Topologies } \\
\cline { 2 - 3 } & Single production & Pair production \\
\hline \hline$L^{ \pm} \rightarrow \nu W$ & - & $\mathrm{h} 210, \mathrm{~h} 400$ \\
$L^{0} \rightarrow \ell W$ & - & $\mathrm{h} 230(\mathrm{~h} 220), \mathrm{h} 420$ \\
\hline \hline$E_{i} \rightarrow \nu_{i} W$ & - & $\mathrm{h} 210, \mathrm{~h} 400$ \\
$N_{i} \rightarrow \ell_{i} W$ & - & $\mathrm{h} 230(\mathrm{~h} 220), \mathrm{h} 420$ \\
\hline \hline$\ell^{*} \rightarrow \ell \gamma$ & $\ell 201(\ell 101)$ & $\ell 202$ \\
$\ell^{*} \rightarrow \nu W$ & $\mathrm{~h} 210(\mathrm{~h} 200), \ell 200$ & $\mathrm{~h} 210, \mathrm{~h} 400$ \\
$\ell^{*} \rightarrow \ell Z$ & $\mathrm{~h} 220(\mathrm{~h} 210), \ell 400$ & - \\
\hline$\nu^{*} \rightarrow \nu \gamma$ & $\ell 001$ & $\ell 002$ \\
$\nu^{*} \rightarrow \ell W$ & $\mathrm{~h} 210(\mathrm{~h} 200), \ell 200$ & $\mathrm{~h} 230(\mathrm{~h} 220), \mathrm{h} 420$ \\
$\nu^{*} \rightarrow \nu Z$ & $\mathrm{~h} 200, \ell 200$ & - \\
\hline \hline$q^{*} \rightarrow q \gamma$ & $\mathrm{h} 201(\mathrm{~h} 101)$ & - \\
$q^{*} \rightarrow q g$ & $\mathrm{~h} 300$ & - \\
\hline \hline$e^{+} e^{-} \rightarrow \gamma \gamma$ & \multicolumn{2}{|c|}{$\ell 002$} \\
\hline
\end{tabular}

Table 3: Observable topologies corresponding to the different production and decay modes of unstable heavy fermions.

The criteria for selecting isolated particles and jet clustering are explained in section 4.1, both for hadronic and leptonic events. As will be seen, in the case of the leptonic events all charged particles are included in the jets and the concept of isolated leptons is not used.

Table 3 shows the relevant topologies for the different production and decay channels. The topologies in brackets do not correspond directly to the physical final state but are often the observed ones. They become particularly important whenever there are particles produced with very low momentum or at small angle to the beam.

Only the topologies that will be considered in this analysis are indicated in the table. Thus in the pair production modes with both heavy leptons decaying into $W$ bosons the topologies corresponding to the purely leptonic decays of the $W W$ pair are not considered, due to their low branching ratio.

Single and double photon final states ( $\ell 001$ and $\ell 002$ topologies) arise in the case of radiatively decaying excited neutrinos. For these topologies, the analyses presented in references 111 are used.

\section{The DELPHI detector and the data samples}

A detailed description of the DELPHI detector and of its performance can be found in [12. This analysis relies both on the charged particle detection provided by the tracking system and on the neutral cluster detection provided by the electromagnetic and hadronic calorimeters.

The main tracking detector of DELPHI is the Time Projection Chamber, which covers the angular range $20^{\circ}<\theta<160^{\circ}$, where $\theta$ is the polar angle defined with respect to 
the beam direction. Other detectors contributing to the track reconstruction are the Vertex Detector, the Inner and Outer Detectors and the Forward Chambers. The best momentum resolution obtained for $45 \mathrm{GeV} / c$ muons is $\sigma(1 / p)=0.57 \times 10^{-3}(\mathrm{GeV} / c)^{-1}$. The VD consists of three cylindrical layers of silicon strip detectors, each layer covering the full azimuthal angle.

Electromagnetic shower reconstruction is performed in DELPHI using the barrel and the forward electromagnetic calorimeters, including the STIC (Small angle TIle Calorimeter), the DELPHI luminosity monitor. The energy resolutions of the barrel and forward electromagnetic calorimeters are parameterized respectively as $\sigma(E) / E=$ $0.043 \oplus 0.32 / \sqrt{E}$ and $\sigma(E) / E=0.03 \oplus 0.12 / \sqrt{E} \oplus 0.11 / E$, where $E$ is expressed in $\mathrm{GeV}$ and the symbol ' $\oplus$ ' implies addition in quadrature. The hadron calorimeter covers both the barrel and forward regions. It has an energy resolution of $\sigma(E) / E=0.21 \oplus 1.12 / \sqrt{E}$ in the barrel.

Photon detection in the region between the barrel and the forward electromagnetic calorimeters (polar angles around $40^{\circ}$ and $140^{\circ}$ ) is achieved using the information of a set of lead/scintillator counters $\left(40^{\circ}\right.$ taggers). The efficiency of the taggers was checked with Bhabha events and found to be greater than $95 \%$.

Finally, muons are identified by their penetration through the iron yoke of the hadron calorimeter to drift chambers covering both the barrel and the forward region of the detector. The barrel region is equipped with three layers of drift chambers, while the end caps contain two planes. One surrounding layer of streamer tubes completes the coverage between the two regions.

The effects of experimental resolution, both on the signals and on backgrounds, were studied by generating Monte Carlo events for the possible signals and for the SM processes, and passing them through the full DELPHI simulation and reconstruction chain. Bhabha events were generated with the Berends, Hollik and Kleiss generator [13], while $e^{+} e^{-} \rightarrow Z \gamma$ events were generated with PYTHIA [14 and KORALZ 15. PYTHIA was also used for the following processes: $e^{+} e^{-} \rightarrow W W, e^{+} e^{-} \rightarrow W e \nu, e^{+} e^{-} \rightarrow Z Z$, and $e^{+} e^{-} \rightarrow Z e e$. In all four-fermion channels, studies with the EXCALIBUR generator [16] were also performed. The two-photon ( " $\gamma \gamma$ ") physics events were generated according to the TWOGAM [17] generator for quark channels and the Berends, Daverveldt and Kleiss generator [18] for the electron, muon and tau channels, and also for the Quark Parton Model giving hadrons. Compton-like final states originating from an $e \gamma$ collision, (with a missing electron in the beam pipe), referred to as Compton events, were generated according to [19], and $e^{+} e^{-} \rightarrow \gamma \gamma$ events according to [20].

Single and pair excited lepton events and single excited quark events were generated according to the cross-sections defined in [6], involving $\gamma$ and $Z$ exchange. Pair production of sequential leptons and non-canonical leptons was generated according to the crosssections given in [3] and [4]. The hadronization and decay processes were simulated by JETSET 7.4 [14]. The initial state radiation effect was included at the level of the generator for single production, while for pair production it was taken into account in the total cross-section. All the expected decay modes were included in the simulation.

\section{Event selection}

The event selection was performed in three stages. In the first level, very general selection criteria were applied and the events were classified according to the topology scheme described above. In the second level, differing selection criteria were applied to each topology. Finally, whenever possible, event flavour tagging was performed, based on the 
identification of the final state leptons and on other (topology dependent) characteristics of the event. Details on each selection level are given below.

\subsection{Basic event selection}

The basic event selection and classification was as follows. Charged particles were considered only if they had momentum greater than $0.1 \mathrm{GeV} / c$ and impact parameters in the transverse plane and in the beam direction below $4 \mathrm{~cm}$ and $10 \mathrm{~cm}$ respectively. Neutral clusters were defined as energy depositions in the calorimeters unassociated with charged particle tracks. All neutrals of energy above $100 \mathrm{MeV}$ were selected.

Visible energy greater than $0.2 \sqrt{s}$ in the polar angle region between $20^{\circ}$ and $160^{\circ}$ was required, including at least one particle with energy greater than $5 \mathrm{GeV}$. Energetic visible particles are expected in all the relevant topologies. Close to the kinematical limit, these particles are produced isotropically. In this way the " $\gamma \gamma$ " background was drastically suppressed since most of the energy in such events is either detected at low polar angles or undetected in the beam pipe.

Events with measured charged or neutral particles having energy greater than $\sqrt{s}$ were rejected. In addition, at least one charged particle in the polar angle region between $25^{\circ}$ and $155^{\circ}$ with associated hits in the Vertex Detector was required. This criterion is useful in rejecting cosmic ray background.

Events with at least six detected charged particles were selected for the hadronic topologies, and those with not more than five for the leptonic topologies.

Charged particles were considered isolated if, in a double cone centred on their track with internal and external half angles of $5^{\circ}$ and $25^{\circ}$, the total energy associated to charged and neutral particles was below $1 \mathrm{GeV}$ and $2 \mathrm{GeV}$ respectively. The energy of the particle was redefined as the sum of the energies contained inside the inner cone. This energy was required to be greater than $4 \mathrm{GeV}$. In all hadronic topologies with isolated leptons, these were required to have associated hits inside a $2^{\circ}$ cone in at least two layers of the Vertex Detector.

Isolated charged particles with an associated electromagnetic energy greater than $20 \%$ of their measured momentum were loosely identified as electrons. In hadronic topologies, they were also required to have an associated hadronic energy lower than $15 \%$ of their measured momentum. Isolated particles were classified as muons by requiring an electromagnetic energy lower than $20 \%$ of their measured momentum and at least one associated hit in the muon chambers.

In both hadronic and leptonic topologies, energy clusters in the electromagnetic calorimeters were considered to be due to photons if there were no tracks associated to them and there were no hits inside a $2^{\circ}$ cone in more than one layer of the Vertex Detector and if at least $90 \%$ of any hadronic energy was deposited in the first layer of the hadron calorimeter.

Photons were considered to be isolated if, in a double cone centred on the cluster and having internal and external half angles of $5^{\circ}$ and $15^{\circ}$, the total energy deposited was less than $1 \mathrm{GeV}$. The energy of the photon was redefined as the sum of the energies of all the particles inside the inner cone and no charged particles above $250 \mathrm{MeV} / \mathrm{c}$ were allowed inside this cone. The photon energy had to be greater than $5 \mathrm{GeV}$. No recovery of converted photons was attempted. In all the studied topologies, photons were required to be above $10^{\circ}$ in polar angle. In addition, for the leptonic topologies the most energetic photon in the event was required to have an energy greater than $10 \mathrm{GeV}$. 
The search for jets in the selected events was performed with the Durham jet algorithm [21]. In this algorithm, a resolution variable

$$
y_{i j}=2 \cdot \frac{\min \left(E_{i}^{2}, E_{j}^{2}\right)}{E_{v i s}^{2}} \cdot\left(1-\cos \theta_{i j}\right)
$$

is computed for all pairs of particles. $E_{i, j}$ are the energies of the particles, $\theta_{i j}$ is their opening angle, and $E_{v i s}$ is the visible energy in the event. The pair for which $y_{i j}$ is smallest is replaced by a pseudoparticle with four-momentum equal to the sum of their four-momenta. In this analysis, the algorithm is used in two different ways:

- the procedure is iterated until all pseudo-particle pairs have $y_{i j}$ larger than a certain $y_{\text {cut }}$ value. A cut-off value of $y_{c u t}=0.003$ was used. This relatively low value of $y_{\text {cut }}$ is well suited for topologies with many jets.

- the procedure is iterated until all particles are clustered into a certain pre-defined number of jets $\left(\mathrm{N}_{\text {jets }}\right)$. In this case the value of $y_{\text {cut }}$ at the last iteration, $y_{\text {cut }\left(N_{\text {jets }}+1 \rightarrow N_{\text {jets }}\right)}$, as well as $y_{\text {cut }\left(N_{\text {jets }} \rightarrow N_{\text {jets }}-1\right)}$, characterize the event topology.

For hadronic events, all neutral and charged particles except isolated leptons and photons were included in the jets. The algorithm was applied four times, requiring $\mathrm{N}_{\text {jets }}=1,2,3$ and 4. In order to increase the purity of the 2-jet event sample, only events with $y_{\text {cut }(3 \rightarrow 2)}<0.06$ and $y_{\text {cut }(2 \rightarrow 1)}>0.001$ were kept. Similarly, for the 3 -jet events the $y_{\text {cut }}$ variables were constrained to $y_{\text {cut }(3 \rightarrow 2)}>0.003$ and $y_{\text {cut }(4 \rightarrow 3)}<0.001$.

For the leptonic events, only the isolated photons were left out of the jets. Charged particles were not treated as isolated objects, but clustered into jets referred to as 'low multiplicity' jets or 'leptonic' jets. This allows for the fact that taus can decay into several charged and neutral particles, and electrons can be accompanied by other electrons and photons due to interactions with matter. In this case the algorithm was applied with $y_{\text {cut }}=0.003$. Whenever the resulting number of jets was lower than the number of isolated leptons previously found $\left(\mathrm{N}_{\text {lept }}\right)$ the algorithm was applied once more imposing $\mathrm{N}_{\text {jets }}=\mathrm{N}_{\text {lept }}$.

The jets in leptonic events were loosely identified as electrons or muons according to the criteria described for isolated leptons. For a jet to be identified as a muon, it was also required not to contain more than two tracks.

Jets were classified as charged if they contained at least one charged particle. In the case of the hadronic (leptonic) topologies, only events with all jets classified as charged and with axes in the polar region between $20^{\circ}\left(25^{\circ}\right)$ and $160^{\circ}\left(155^{\circ}\right)$ were retained.

\subsection{Selected hadronic events}

\subsubsection{Single production topologies}

In hadronic events from single heavy lepton production, the jets originate from the decay of a $W$ or a $Z$ which is not produced at rest. The candidates must have two charged jets with high acollinearity $\left(A_{\text {col }}^{j j}\right)$ and acoplanarity $\left(A_{c o p}^{j j}\right)$ [', and the 2-jet system must have a high mass $\left(\mathrm{M}_{j j}\right)$ and a high momentum $\left(P_{j j}\right)$. The main backgrounds for these topologies are $e^{+} e^{-} \rightarrow q \bar{q}(\gamma)$ events, including radiative returns to the $Z\left(e^{+} e^{-} \rightarrow Z \gamma\right)$ where the photon was lost in the beam pipe, and semileptonic decays of $W$ pairs. In the first case the events are characterized by two acollinear jets. In addition, radiative return events have a high missing momentum $(\not p)$ at low polar angle $(\not \partial)$. The semileptonic

\footnotetext{
${ }^{3}$ The acoplanarity is defined as the acollinearity in the plane perpendicular to the beam.
} 
$W W$ events are characterized by the fact that the mass recoiling against the 2-jet system $\left(M_{R}\right)$ should be close to the $W$ mass, which is not true for the signal. Fully hadronic $W W$ events rejection can be achieved by cutting tighter in the event topology variable $y_{\text {cut }(3 \rightarrow 2)}$.

\begin{tabular}{|c||c|c|c|c|}
\hline \multirow{2}{*}{ Topol } & \multicolumn{4}{c|}{ Selection variables } \\
\cline { 2 - 5 } & angles & masses & other criteria & fit \\
\hline \hline $\mathrm{h}$ 300 & $\min ^{\mathrm{A}}\left(\mathrm{A}^{j j}\right)>40^{\circ}$ & & & $4 \mathrm{C}$ \\
\hline $\mathrm{h} \mathbf{2 0 0}$ & $\mathrm{A}_{\text {col }}^{j j}>40^{\circ}$ & $\mathrm{M}_{j j} \in[40,100] \mathrm{GeV} / c^{2}$ & $y_{\text {cut }(3 \rightarrow 2)}<0.01$ & $1 \mathrm{C}$ \\
& $\mathrm{A}_{\text {cop }}^{j j}>25^{\circ}$ & $\mathrm{M}_{R}<60 \mathrm{GeV} / c^{2}$ if $\mathbf{W}$ & $\not p>0.11 \sqrt{s}$ & \\
& $\theta^{\circ}>25^{\circ}$ & $\mathrm{M}_{R}<75 \mathrm{GeV} / c^{2}$ if $\mathbf{Z}$ & & \\
\hline $\mathrm{h} \mathbf{2 1 0}$ & $\mathrm{A}_{\text {col }}^{j j}>30^{\circ}$ & $\mathrm{M}_{j j}>40 \mathrm{GeV} / c^{2}$ & & $1 \mathrm{C}$ \\
& $\mathrm{A}_{\text {cop }}^{j j}>15^{\circ}$ & $\mathrm{M}_{R}<60 \mathrm{GeV} / c^{2}$ if $\mathbf{W}$ & & \\
& $\theta^{\circ}>20^{\circ}$ & $\mathrm{M}_{R}<75 \mathrm{GeV} / c^{2}$ if $\mathbf{Z}$ & & \\
\hline $\mathrm{h} \mathbf{2 2 0}$ & $\mathrm{A}_{\text {col }}^{j j}>20^{\circ}$ & & & $5 \mathrm{C}$ \\
& $\mathrm{A}_{\text {cop }}^{j j}>10^{\circ}$ & & & $1 \mathrm{C}$ \\
& $\theta^{\circ}>20^{\circ}$ & & $E_{\gamma}>20 \mathrm{GeV}$ & $4 \mathrm{C}$ \\
\hline $\mathrm{h} \mathbf{2 0 1}$ & $\mathrm{A}_{i s o}^{\gamma}>25^{\circ}$ & & $E_{\gamma}>30 \mathrm{GeV}$ & $4 \mathrm{C}$ \\
& $\theta_{\gamma}^{\gamma}>40^{\circ}$ & & & \\
\hline $\mathrm{h} \mathbf{1 0 1}$ & $\mathrm{A}_{\text {col }}^{j \gamma}<30^{\circ}$ & & & \\
& $\theta_{\gamma}>40^{\circ}$ & & & \\
\hline
\end{tabular}

Table 4: Selection criteria for single production hadronic topologies. The notation for the topologies is the one defined in section 2.4.

Hadronic events from single excited quark production are characterized by the presence of three jets (h300) in the case of the decay through the gluon and by one energetic photon with a large isolation angle $\left(\mathrm{A}_{\text {iso }}^{\gamma}\right)$ and two jets (h201) in the case of the electromagnetic radiative decay. The 3 -jet topologies were selected using the event topology variable $y_{\text {cut }(3 \rightarrow 2)}$ and the minimum angle between jets, $\min \left(A^{j j}\right)$. The photon is expected to have a rather large energy $\left(E_{\gamma}\right)$ and an isotropic polar angle distribution $\left(\theta_{\gamma}\right)$. The main backgrounds for these topologies are $e^{+} e^{-} \rightarrow q \bar{q}(\gamma)$ events and hadronic decays of $W$ pairs. Near the kinematic limit the spectator quark is essentially produced at rest and the observed topologies are then h200 and h101. While the h101 corresponds to a very clear signature, in the h200 the SM $q \bar{q}$ events constitute an enormous and irreducible background.

In order to improve the estimation of the momentum and energy of the jets, a kinematic constrained fit was applied to the selected events. The constraints imposed depend on the particular final state being studied. In several of the relevant hadronic final states, jet pairs come from the decay of $\mathrm{W}$ or $\mathrm{Z}$ bosons. Therefore, the invariant mass of the two-jet system can be required to be either $m_{W}$ or $m_{Z}$. Since the h200 and h210 topologies can arise from both the $W$ and the $Z$ channels (see tables 2 and 3) the fit was performed twice for these topologies, using $m_{W}$ and $m_{Z}$. If there are no undetected particles, energy and momentum conservation can be imposed. This is the case for the topologies with isolated photons and for the h300 topology (4C fit). For the h220 topology the fit was performed both requiring only the invariant mass of the two-jet system to be $m_{Z}(1 \mathrm{C})$ and imposing the additional constraint of energy-momentum conservation $(5 \mathrm{C})$. The input quantities for the fit are basically the measured energies and momenta of the objects (particles 
or jets). Lagrange multipliers are used to make a constrained fit to both the energies and the directions of the jets and isolated particles. The fit requires a $\chi^{2}$ value to be optimized, subject to the given constraints on the reconstructed final state objects. The details of the fitting procedure, including the errors on the input variables, can be found in reference [22].

The main selection criteria for the different topologies are summarized in table $₫$. The number of constraints imposed for each topology is also given in the table. In all cases, only events with a $\chi^{2}$ per degree of freedom lower than 5 were retained. Whenever a topology was relevant for more than one search channel, some of the selection criteria could vary from one case to the other. This is indicated in table 1 for the recoil mass cut in the case of the h200 and h210 topologies.

The selection criteria allow quite an efficient background rejection. The cut values were tuned for each topology in order to optimize the signal to noise ratio. For the h200 topology, events with signals in more than two $40^{\circ}$ taggers inside a $20^{\circ}$ cone centred on the direction of the missing momentum were rejected. This criterion is useful in rejecting $q \bar{q} \gamma$ events in which the photon was lost in the region between the electromagnetic calorimeters.

In all the topologies with two jets in the final state, events were required to have a charged multiplicity of at least eight.

\subsubsection{Pair production topologies}

The relevant topologies for the pair production of charged heavy leptons can have two jets and one lepton or four jets, resulting from the decay of the two $W$ s (see table 2). As mentioned, fully leptonic decay modes, with their rather small branching ratios, will not be considered in this analysis. The main topologies are thus h210 and h400.

\begin{tabular}{|c|c|c|c|c|c|}
\hline \multirow{2}{*}{$\begin{array}{l}\text { Heavy } \\
\text { lepton }\end{array}$} & \multirow{2}{*}{$\begin{array}{l}\text { Topo- } \\
\text { logy }\end{array}$} & \multicolumn{4}{|c|}{ Selection variables } \\
\hline & & leptons & $\mathrm{y}_{\text {cut }}$ & missing & other criteria \\
\hline Neutral & $\begin{array}{l}\text { h230 \& } \\
\text { h420 }\end{array}$ & $N_{\text {lept }} \geq 2$ & $\overline{y_{\text {cut }(2 \rightarrow 1)}>0.03}$ & $\theta \theta^{\prime}>20^{\circ}$ & \\
\hline \multirow[t]{2}{*}{ Charged } & h210 & $N_{\text {lept }}>0$ & & $\begin{array}{c}\theta>25^{\circ} \\
E{ }^{\prime}>0.5 \sqrt{s} \\
\not p_{T}>10 \mathrm{GeV} / c\end{array}$ & $\begin{array}{l}E_{m i s}^{15}<0.5 \\
A_{\text {lept }}^{i s o}>50^{\circ}\end{array}$ \\
\hline & h400 & $N_{\text {lept }}=0$ & $\begin{array}{c}y_{\text {cut }(3 \rightarrow 2)}>0.03 \\
y_{\text {cut }(4 \rightarrow 3)}>0.003\end{array}$ & $\begin{array}{c}\theta^{\prime}>30^{\circ} \\
E>0.3 \sqrt{s}\end{array}$ & $\begin{array}{c}\mathrm{A}^{W W}<140^{\circ} \\
\not p / \not E<0.7\end{array}$ \\
\hline
\end{tabular}

Table 5: Selection criteria for pair production hadronic topologies.

In the case of neutral heavy leptons, two additional charged leptons are present in the final state, and the main topologies are, thus, h230 and h420. All hadronic events with at least two isolated leptons found were considered.

The additional final state leptons present in signal events for neutral heavy lepton production constitute a rather clear signature. On the contrary, for the charged heavy leptons search channels there is a nearly irreducible background from $W W$ events. Signal events are characterized by the presence of two additional neutrinos, seen as additional missing energy. The missing momentum will in general be neither forward nor aligned with the directions of the jets. Thus, missing energy $(E)$ and transverse missing momen- 
tum $\left(\not p_{T}\right)$ are expected and the energy in a $15^{\circ}$ cone around the direction of the missing momentum $\left(E_{m i s}^{15}\right)$ will be low.

In the final state topology with no isolated leptons (h400), $q q(\gamma)$ background can be rejected using the $y_{\text {cut }}$ variables to select 4 -jet events. In this 4 -jet topology, the two $W$ candidates (i.e. the two jet pairs supposed to result from the decay of the $W$ bosons) were found by trying all the possible combinations and choosing the one for which the jet-jet invariant masses best reproduce the $W$ mass. The angle between the two $\mathrm{W}$ candidates $\left(A^{W W}\right)$ is expected to be close to $180^{\circ}$ for the $W W$ background and lower for the signal, due to the presence of the two additional neutrinos. In the final state with one isolated lepton (h210), it is also required that the lepton is well isolated $\left(A_{\text {lept }}^{\text {iso }}\right)$. The selection criteria applied in the search for both neutral and charged pair-produced heavy leptons are summarized in table 5.

\subsection{Selected leptonic events}

Events classified as leptonic can originate from radiative decays of heavy leptons, in which case there will be photons in the final state, or from decays into $W$ or $Z$ bosons decaying into leptons, in which case there will be only leptonic jets involved (see table 2 and table 3). The two analyses are quite different and will be treated separately.

\subsubsection{Leptonic events without isolated photons}

The topologies considered in this section are $\ell 200$, two low multiplicity jets only, and $\ell 400$, four low multiplicity jets. As mentioned, they arise whenever there are $W$ or $Z$ bosons decaying leptonically. Since these topologies arise only in single production modes, they have to be considered in the search for excited leptons only.

\section{$\ell 200$ topology}

The signal events are characterized by the presence of two acoplanar leptonic jets and missing energy. The background for this topology comes essentially from $e^{+} e^{-} \rightarrow \ell^{+} \ell^{-}(\gamma)$ processes, in particular Bhabha events where the photon is lost, and from leptonic decays of $W$ pairs. In the $W W$ background events, both the leptonic jets come from $W$ decays, having a large momentum. The general selection criteria were the following:

- $\mathrm{A}_{\text {col }}>10^{\circ}$

- $\mathrm{A}_{\text {cop }}>10^{\circ}$

- $\not p>0.11 \sqrt{s}$

- $\theta>30^{\circ}$ for both leptonic jets.

As seen in table 3, the topology $\ell 200$ can arise in several different channels. In addition to the general selection criteria for the topology, a different specific cut was included for each decay channel. This cut depends on the origin of the leptonic jets present in the final state. They can be spectator leptons produced together with the heavy one, products of the decay of the heavy lepton or products of the decay of a $W$ or $Z$ boson.

If the decaying excited lepton is charged, $\ell \ell^{*} \rightarrow(\ell \nu W, \ell \ell Z) \rightarrow \ell \ell \nu \nu$, the lower energy leptonic jet is expected to be the spectator lepton and the momentum of the least energetic charged jet was required to be lower than $0.11 \sqrt{s}$.

For excited neutrinos, in the case of the decay via a $W\left(\nu \nu^{*} \rightarrow \nu \ell W \rightarrow \ell \ell \nu \nu\right)$ the mass recoiling against one of the two leptonic jets was required to be in the $\mathrm{W}$ mass region $\left(70 \mathrm{GeV} / c^{2}<m<110 \mathrm{GeV} / c^{2}\right)$, while in the case of the decays via a $Z\left(\nu \nu^{*} \rightarrow \nu \nu Z \rightarrow\right.$ 
$\nu \nu \ell \ell)$ the invariant mass of the two leptonic jets had to be between $80 \mathrm{GeV} / c^{2}$ and 100 $\mathrm{GeV} / c^{2}$.

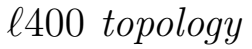

This topology can arise in the case of a singly produced charged excited lepton decaying via a $Z$ boson. For signal events, two of the leptonic jets result from the $Z$ decay and thus have a large invariant mass, while the second pair of leptonic jets has in general a low invariant mass.

The background for this topology comes from 4-fermion processes. In this analysis it was required that at least one of the leptonic jets had been previously identified as an isolated lepton (see section 4.1) to avoid hadronic contamination.

\subsubsection{Leptonic events with isolated photons}

Final states resulting from singly produced charged excited leptons decaying radiatively are characterized by an energetic photon $\left(\mathrm{E}_{\gamma}\right)$ in the central region of the detector and two low multiplicity jets ( $\ell 201$ topology). Near the kinematic limit one of these jets will not be observed due to its low momentum ( $\ell 101$ topology). This final state topology is particularly relevant when the t-channel cross-section dominates ( $e^{*}$ single production) and the spectator lepton is frequently lost in the beam pipe.

The main background for the $\ell 201$ topology comes from $e^{+} e^{-} \rightarrow \ell^{+} \ell^{-}$radiative events. Events having a photon emitted at very low polar angle $\left(\theta_{\gamma}\right)$ or with low energy are easily eliminated. However, events with a hard isolated photon constitute an irreducible background.

For the $\ell 101$ topology, the background comes from Bhabha events where one electron was lost or misidentified as a photon, and from Compton events. In Bhabha events, there are two essentially back-to-back particles in the forward regions of the detector, i.e., the jet-photon space angle $\left(\mathrm{A}^{j \gamma}\right)$ is around $180^{\circ}$. In Compton events the charged jet and the photon are acollinear and there is a large amount of energy deposited at relatively low polar angles.

Final states resulting from pair-produced charged excited leptons decaying radiatively are characterized by the presence of two leptonic jets and two hard photons in the detector. Possible background comes from doubly radiative $e^{+} e^{-} \rightarrow \ell^{+} \ell^{-}$events. In signal events there must be two lepton-photon combinations with compatible invariant masses, which correspond to the excited lepton invariant mass. The relevant variable is the minimum of the lepton-photon invariant mass differences: $\Delta m_{\ell \gamma}=\min \left(\left|m_{\ell 1 \gamma 1}-m_{\ell 2 \gamma 2}\right|, \mid m_{\ell 1 \gamma 2}-\right.$ $\left.m_{\ell 2 \gamma 1} \mid\right)$.

In three- or four-body topologies, the energies can be rescaled by imposing energy and momentum conservation and using just the polar and azimuthal angles, which are well measured in the detector. This procedure can significantly improve the energy resolution. The compatibility of the momenta calculated from the angles with the measured momenta was quantified on a $\chi^{2}$ basis.f. For two-body topologies, the same method can be applied assuming the presence of a third particle along the beam direction.

Only events with $\chi^{2}<5$ either for photons or for charged particles were kept. The fact that the condition $\chi^{2}<5$ was not applied simultaneously to the photons and to the charged particles allows events in the electron and muon channels with photons

\footnotetext{
${ }^{4}$ The $\chi^{2} \quad$ parameter was defined separately for charged jets $\left(\chi_{\text {charged }}^{2}\right)$ and photons $\left(\chi_{\text {photons }}^{2}\right)$ as $\chi^{2}=\frac{1}{n} \sum_{i=1, n}\left(\frac{p_{i}^{\text {calc }}-p_{i}^{\text {meas }}}{\sigma_{i}}\right)^{2}$ where $n$ is the number of measured particles, $p_{i}^{\text {meas }}$ are the measured momenta or energies and $p_{i}^{\text {calc }}$ are the momenta calculated from the kinematic constraints. $\sigma_{i}$, the quadratic sum of the errors on $p_{i}^{\text {calc }}$ and $p_{i}^{\text {meas }}$, is defined in reference 10
} 
near the boundaries of the calorimeter modules (where electromagnetic energy can be badly reconstructed) to be kept. The rescaling procedure can also be applied to the tau channel because the charged decay products nearly follow the direction of the primary tau. However, due to the neutrinos, the rescaled momenta of the charged jets are expected to be substantially different from the measured ones. This can be used in the tau lepton identification through a cut $\chi_{\text {charged }}^{2}>5$. In addition to this criteria, the events selected in 3-body topologies were checked for their coplanarity (the sum of the angles between the particles had to be greater than $355^{\circ}$ ).

\begin{tabular}{|c|c|c|c|}
\hline \multirow[b]{2}{*}{ Topology } & \multicolumn{3}{|c|}{ Selection variables } \\
\hline & jet variables & photon variables & other \\
\hline$\ell \ell 201 e$ & \multirow{2}{*}{$\overline{\mathrm{p}_{\text {jet }_{1}}>10 \mathrm{GeV} / \mathrm{c}}$} & $\begin{array}{l}\theta_{\gamma}>40^{\circ} \\
\end{array}$ & \\
\hline$\ell 201 \mu$ & & & \\
\hline$\ell 201 \tau$ & & $\theta_{\gamma}>20^{\circ}$ & \\
\hline$\ell 101 e$ & \multirow{3}{*}{$\mathrm{p}_{\text {jet }}>10 \mathrm{GeV} / \mathrm{c}$} & \multirow{3}{*}{$\begin{array}{c}\mathrm{E}_{\gamma}>0.22 \sqrt{s} \\
\theta_{\gamma}>40^{\circ}\end{array}$} & $100^{\circ}<\mathrm{A}^{j \gamma}<179^{\circ}$ \\
\hline$\ell 101 \mu$ & & & \\
\hline$\ell 101 \tau$ & & & $100^{\circ}<\mathrm{A}^{j \gamma}<179^{\circ}$ \\
\hline$\ell 202 e$ & \multirow{3}{*}{$\begin{array}{l}\mathrm{p}_{\text {jet }_{1}}>10 \mathrm{GeV} / c \\
\mathrm{p}_{\text {jet }_{2}}>10 \mathrm{GeV} / c\end{array}$} & & $\Delta m_{\ell \gamma}<15 \mathrm{GeV} / c^{2}$ \\
\hline$\ell 202 \mu$ & & & $\Delta m_{\ell \gamma}<10 \mathrm{GeV} / c^{2}$ \\
\hline$\ell 202 \tau$ & & & $\Delta m_{\ell \gamma}<20 \mathrm{GeV} / c^{2}$ \\
\hline
\end{tabular}

Table 6: Selection criteria for leptonic topologies with isolated photons. The topologies below the double line correspond to the pair production.

The backgrounds and efficiencies depend significantly on the flavour of the final state leptons involved in these topologies. Thus, different selection criteria are used for the different flavours. The main selection criteria are summarized in table 6 .

For the $\ell 101$ topology, an additional cut was applied in the electron channel: events that have a rescaled momentum greater than $0.18 \sqrt{s}$ assigned to the particle lost along the beam direction were rejected. This criterion is useful to eliminate Compton events.

\subsection{Events with photons only}

Neutral excited leptons can give rise to single or double photon events. Thus, excited neutrinos produced in pairs and decaying radiatively to an ordinary neutrino would be tagged through the $\ell 002$ topology and those produced singly would be detected through the $\ell 001$ topology. For these topologies, the analyses described in [11] are used.

The SM processes constituting the background to both topologies are essentially QED $e^{+} e^{-} \rightarrow \gamma \gamma, e^{+} e^{-} \rightarrow Z \gamma(\gamma)$ with $Z \rightarrow \nu \nu$, radiative Bhabhas and Compton events.

In order to reduce drastically the Bhabha and Compton background, single gamma events were required to have polar angles above $45^{\circ}$ and no other electromagnetic energy deposition was allowed out of a $20^{\circ}$ cone. Furthermore, it was required that the photons had a line of flight compatible within $15^{\circ}$ with the shower direction reconstructed by the HPC calorimeter. This criterion and an additional selection based on the HCAL were applied to veto cosmics.

The two-photon sample was selected requiring at least two photons satisfying the following criteria: 
- Energy greater than $25 \%$ of the centre-of-mass energy in the polar region between $25^{\circ}$ and $155^{\circ}$.

- At least three HPC layers with more than $5 \%$ of the total electromagnetic energy, for HPC energy depositions not pointing to the $\phi$ intermodular zones.

Furthermore, the hadronic energy was required to be less than $15 \%$ of the total deposited energy unless the photon fell in the HPC $\phi$ cracks, in which case it was required that the HCAL first layer energy deposition was greater than $90 \%$ of the total hadronic energy.

The $\gamma \gamma$ sample was enriched through the recovery of photons converted after the VD detector. The number of converted photons was limited to one per event and their recovering was performed in a slightly reduced geometrical acceptance $\left(\theta>30^{\circ}\right)$, in order to keep a high level of background rejection. A converted photon was defined as an energy deposition associated to a charged particle track and with no VD track elements within $2^{\circ}$ and $6^{\circ}$ for the barrel $\left(\theta>40^{\circ}\right)$ and the forward $\left(\theta<35^{\circ}\right)$ regions, respectively. A VD track element was defined by at least two $R \phi$ hits on different layers within a tolerance of 0.5 degrees.

\subsection{Event flavour identification}

The event flavour, in the hadronic topologies with isolated leptons, was tagged by loosely identifying the leptons according to the criteria described in section 4.1: in the h210 topology, events were tagged as electronic (muonic) events if the final state lepton was identified as an electron (muon) and in h220 if one of the final state leptons was identified as an electron (muon) and the other one was not identified as a muon (electron). In the tau channel, the momentum of the isolated lepton is expected to be lower than for the other leptonic flavours. For the h220 topology the lower energy lepton is expected to be the spectator lepton produced together with the excited one and in the tau channel it was required that $\mathrm{p}_{\ell}<0.11 \sqrt{s}$. The same is true for the h210 topology when it arises from the $W$ decay of a charged excited lepton $\left(\ell \ell^{*} \rightarrow \ell \nu W\right)$. In other cases, such as for neutral excited leptons, the final state lepton can be more energetic and in the tau channel it was required that $\mathrm{p}_{\ell}<0.22 \sqrt{s}$.

In the topologies corresponding to the pair production of neutral heavy leptons, all events with more than two isolated leptons in the final state were kept. If only two leptons were present, events were kept as candidates in the electron (muon) channel if both leptons were identified as such.

The flavour identification for leptonic topologies was performed using the leptonic jet identification (see section 4.1) and, whenever possible, the comparison between the measured momenta and the momenta computed using the rescaling procedure (see section 4.3.2).

In the leptonic topologies with no isolated photons, the rescaling procedure was not applied, since there are always at least two neutrinos involved. There is also at least one charged jet coming from the decay of a $W$ or $Z$ boson and containing no relevant flavour information. Events were classified as electron or muon events whenever the lowest energy jet was identified as such. All events were kept in the tau channel.

In the topologies involving isolated photons, since there are no missing particles or there is only one particle lost along the beam pipe, the momenta can be computed imposing energy-momentum conservation. Events were kept as candidates in the electron (muon) channel if at least one of the jets was identified as an electron (muon) and no jets were identified as muons (electrons) and if $\chi_{\text {charged }}^{2}<5$. For the $\ell 101$ topology, where 
background problems are more severe, it was also required that $\chi_{\text {photons }}^{2}<5$. Events were kept as candidates in the tau channel if $\chi_{\text {charged }}^{2}>5$ and $\chi_{\text {photons }}^{2}<5$.

\section{Results}

The number of candidates at different selection levels are given in table ?? for the hadronic topology, and in tables ?? and ?? for the leptonic topology with (table ??) and without (table ??) isolated photons. Selection level 1 corresponds to the general criteria, before any specific topology cuts (section 4.1). Level 2 corresponds to specific topology cuts, without flavour tagging (sections 4.2 and 4.3). Flavour tagging is included in level 3 (section 4.5). The numbers in brackets give the simulated SM background expectations. The topologies marked with a $\mathrm{P}$ correspond to the pair production modes. Whenever a topology is relevant for both the charged and the neutral new leptons search and different selection criteria were applied (see sections 4.2.1 and 4.3.1), the name of the topology is followed by 'char' or 'neut'. A given selection level is always a subsample of the previous one. The different flavours considered at a given level are not exclusive. In the different selection levels and topologies, fair agreement between data and the SM expectation is found.

Data and SM simulation distributions at $\sqrt{s}=183 \mathrm{GeV}$ for the hadronic topologies at selection level 1 are shown in figures 11 and 2. Figure 1(a) and (b) show the jet-jet acollinearity and the jet-jet invariant mass for the h200 and h201 topologies respectively. In 1(c) the momentum of the lepton in the h210 topology is shown. Figure 1)(d) concerns the h400 topology and shows the angle between the two jet-pairs taken as $W$ candidates. There is, for all the distributions, a fair overall agreement. As will be discussed below, the distributions shown in figure 2 are the ones relevant for signal mass reconstruction. The presence of a signal would correspond to a peak in these variables. As before, there is reasonable overall agreement and no relevant signal is observed. It should be noted that in (a) and (b) there are three and two entries per event respectively corresponding to the different possible jet-jet and jet-photon combinations.

Figure 3 shows distributions for the $\ell 201$ topology at the first level of the event selection for $\sqrt{s}=183 \mathrm{GeV}$. Figure 3 (a) shows the invariant mass for the lepton-lepton pairs using the momenta calculated from the kinematic constraints. The two possible $\ell \gamma$ invariant mass combinations are plotted in figure 3(b). Figures 3(c) and 3(d) display the energy and the isolation angle of the radiated photon. There is reasonable agreement between the data and SM simulation.

In many topologies, the heavy fermion mass can be estimated from the momenta and directions of final state particles. Relevant cases are the $\ell \gamma$ invariant mass for radiatively decaying excited leptons, the jet- $\gamma$ and jet-jet invariant masses for excited quarks, the jet-jet-lepton invariant mass and the recoil mass of isolated leptons for the situations involving $W$ and $Z$ bosons. Signal simulation studies allowed the determination of the mass resolution for each situation. In leptonic events, the mass resolution on the leptonphoton invariant mass, after applying the kinematic constraints, was found to be about $1 \mathrm{GeV} / c^{2}$ for muons, $1.5 \mathrm{GeV} / c^{2}$ for electrons and $2 \mathrm{GeV} / c^{2}$ for taus. In the $\mathrm{h} 300$ and h201 topologies, the resolution on the jet-jet and jet-photon invariant masses after the kinematic fits was found to be about $2 \mathrm{GeV} / c^{2}$. For the h101 topology no kinematic fit was applied and the resolution was around $20 \mathrm{GeV} / c^{2}$. In hadronic events with isolated leptons, the resolution on the lepton recoil mass $\left(m_{\ell}^{2}=s-2 k P_{\ell} \sqrt{s}\right.$, where $k=1.0$ for electrons and muons and $k=1.4$ for taus to take in account the missing energy in the tau decay) is about $1 \mathrm{GeV} / c^{2}$ for muons, $3 \mathrm{GeV} / c^{2}$ for electrons and $5 \mathrm{GeV} / c^{2}$ for 
taus. The resolution on the jet-jet-lepton invariant mass is about $5 \mathrm{GeV} / c^{2}$ for muons and $8 \mathrm{GeV} / c^{2}$ for electrons. In this case, no mass reconstruction was attempted in the tau channel.

The results for single and double photon final state are as follows. In the single photon channel, three events having a single $\gamma$ in the barrel region (i.e. $\theta_{\gamma}=45^{\circ}-135^{\circ}$ ) with $E_{\gamma}>80 \mathrm{GeV}$ were found at $\sqrt{s}=183 \mathrm{GeV}$, while 2.5 were expected from the SM reaction $e^{+} e^{-} \rightarrow \gamma \nu \bar{\nu}$. At $\sqrt{s}=172$ and $\sqrt{s}=161 \mathrm{GeV}$, no events were found with $E_{\gamma}>75 \mathrm{GeV}$ and $E_{\gamma}>70 \mathrm{GeV}$ respectively, while $0.02 \pm 0.01$ and $0.08 \pm 0.03$ were expected from the simulation.

In the two photon channel four events with an acoplanarity greater than $10^{\circ}$ were found at $\sqrt{s}=183 \mathrm{GeV}$, while $0.4 \pm 0.1$ events were expected from the QED background reaction $e^{+} e^{-} \rightarrow \gamma \gamma$ and $1.5 \pm 0.2$ from the process $e^{+} e^{-} \rightarrow Z \gamma \gamma$ with the $Z$ decaying into neutrinos. Two events with an acoplanarity greater than $10^{\circ}$ were found at $\sqrt{s}=172$ $\mathrm{GeV}$, while $0.09 \pm 0.02$ were expected from $e^{+} e^{-} \rightarrow \gamma \gamma$ and $0.61 \pm 0.02$ from $e^{+} e^{-} \rightarrow$ $Z \gamma \gamma \rightarrow \nu \nu \gamma \gamma$. No candidates were found at $\sqrt{s}=161 \mathrm{GeV}$ while $0.8 \pm 0.1$ were expected.

\section{Limits}

The search for the production of unstable heavy fermions involves many final states. The relevance of the different final states depends, as discussed in section 2.3, on the decay branching ratios which are a function of the heavy fermion mass and of the coupling parameters.

The numbers of excited fermion candidates in the single production topologies, as well as the SM expectations, are summarized in table 7 for the different excited fermion types and decay modes and for the three centre-of-mass energies. It should be noted that these numbers result from the combination of the different topologies (tables ??, ?? and ??) and there is, in many cases, overlap between the candidates selected in the different decay channels listed in table 7 .

For exotic leptons only pair production was considered. The number of heavy lepton candidates found and the SM simulation expectations at $\sqrt{s}=183,172$ and $161 \mathrm{GeV}$ are summarized in table 8 for pair production modes.

The possible heavy fermion masses can be deduced in many of the topologies, as referred to in the previous section. Events for which the mass could not be estimated were treated as candidates for all possible mass values.

The efficiencies, including the trigger efficiency, are given in table 9 for all the studied channels and for chosen heavy fermion mass values at $\sqrt{s}=183 \mathrm{GeV}$. The efficiency levels are very similar for scaled masses at the other centre-of-mass energies. The trigger efficiency was estimated to be greater than $85 \%$ and $95 \%$ for the single and the double photon channels respectively. For all the other topologies it is greater then $99 \%$ (being essentially $100 \%$ for all hadronic topologies). The dependence of the efficiency on the mass is weak, due to the combination of the several topologies and of the different centreof-mass energies.

The limits were computed using the method described in [23]. This is the method used in the DELPHI Higgs search analysis, and is well suited both for the combination of channels and for the inclusion of mass information. Each topology at each centre-of-mass energy was treated as a channel, and the used mass resolution depends on the specific reconstruction procedure for each topology, as explained in the previous section.

For the single production of excited fermions, the production cross section is a function not only of the mass of the particle but also of the ratio of the coupling of the excited 


\begin{tabular}{|c|c|c|c|c|}
\hline$\sqrt{s}$ & channel & $e$ & $\mu$ & $\tau$ \\
\hline \multirow{8}{*}{$\begin{array}{c}183 \\
\mathrm{GeV}\end{array}$} & $\ell^{*} \rightarrow \ell \gamma$ & $\overline{444(53 \pm 4)}$ & $\overline{17(16 \pm 1)}$ & $21(22 \pm 2)$ \\
\hline & $\ell^{*} \rightarrow \nu W$ & $17(16 \pm 2)$ & $7(8 \pm 1)$ & $22(17 \pm 2)$ \\
\hline & $\ell^{*} \rightarrow \ell Z$ & $26(31 \pm 2)$ & $22(17 \pm 1)$ & $46(41 \pm 3)$ \\
\hline & $\nu^{*} \rightarrow \nu \gamma$ & \multicolumn{3}{|c|}{$3(2.5 \pm 0.3)$} \\
\hline & $\nu^{*} \rightarrow \ell W$ & $17(18 \pm 2)$ & $10(12 \pm 2)$ & $26(25 \pm 2)$ \\
\hline & $\nu^{*} \rightarrow \nu Z$ & \multicolumn{3}{|c|}{$15(12 \pm 1)$} \\
\hline & $q^{*} \rightarrow q \gamma$ & \multicolumn{3}{|c|}{$120(114 \pm 5)$} \\
\hline & $q^{*} \rightarrow q g$ & \multicolumn{3}{|c|}{$84(98 \pm 5)$} \\
\hline \multirow{8}{*}{$\begin{array}{c}172 \\
\mathrm{GeV}\end{array}$} & $\ell^{*} \rightarrow \ell \gamma$ & $8(9 \pm 1)$ & $7(4.8 \pm 0.4)$ & $5(5.2 \pm 0.8)$ \\
\hline & $\ell^{*} \rightarrow \nu W$ & $2(3.6 \pm 0.5)$ & $1(1.9 \pm 0.3)$ & $1(4.2 \pm 0.4)$ \\
\hline & $\ell^{*} \rightarrow \ell Z$ & $4(4.9 \pm 0.5)$ & $3(2.5 \pm 0.3)$ & $7(7.3 \pm 0.6)$ \\
\hline & $\nu^{*} \rightarrow \nu \gamma$ & \multicolumn{3}{|c|}{$0(0.02 \pm 0.01)$} \\
\hline & $\nu^{*} \rightarrow \ell W$ & $4(3.7 \pm 0.4)$ & $3(2.1 \pm 0.4)$ & $7(5.5 \pm 0.5)$ \\
\hline & $\nu^{*} \rightarrow \nu Z$ & \multicolumn{3}{|c|}{$3(2.7 \pm 0.4)$} \\
\hline & $q^{*} \rightarrow q \gamma$ & \multicolumn{3}{|c|}{$36(33 \pm 2)$} \\
\hline & $q^{*} \rightarrow q g$ & \multicolumn{3}{|c|}{$27(24 \pm 1)$} \\
\hline \multirow{8}{*}{$\begin{array}{c}161 \\
\mathrm{GeV}\end{array}$} & $\ell^{*} \rightarrow \ell \gamma$ & $5(15 \pm 1)$ & $6(7 \pm 1)$ & $10(7 \pm 1)$ \\
\hline & $\ell^{*} \rightarrow \nu W$ & $2(3.2 \pm 0.4)$ & $4(1.8 \pm 0.2)$ & $4(2.9 \pm 0.3)$ \\
\hline & $\ell^{*} \rightarrow \ell Z$ & $1(2.9 \pm 0.4)$ & $2(1.2 \pm 0.2)$ & $7(4.0 \pm 0.4)$ \\
\hline & $\nu^{*} \rightarrow \nu \gamma$ & \multicolumn{3}{|c|}{-} \\
\hline & $\nu^{*} \rightarrow \ell W$ & $1(3.2 \pm 0.4)$ & $2(1.3 \pm 0.2)$ & $7(4.5 \pm 0.5)$ \\
\hline & $\nu^{*} \rightarrow \nu Z$ & \multicolumn{3}{|c|}{$2(1.4 \pm) 0.3$} \\
\hline & $q^{*} \rightarrow q \gamma$ & \multicolumn{3}{|c|}{$40(44 \pm 3)$} \\
\hline & $q^{*} \rightarrow q g$ & \multicolumn{3}{|c|}{$29(30 \pm 2)$} \\
\hline
\end{tabular}

Table 7: Number of excited fermion candidates for the different decay channels and the different centre-of-mass energies in the single production mode. The numbers in brackets correspond to the simulated SM background expectations.

lepton to its mass. 95\% confidence level (CL) upper limits on the ratio $\lambda / m_{f^{*}}$ (see section 2.3) as a function of the $f^{*}$ mass were derived. Figures 1 and 5 show these limits for the excited leptons assuming $f=f^{\prime}$ and $f=-f^{\prime}$ respectively.

Figures 6(a) and 6(b) show the limits on the single production of excited quarks, namely limits on $\lambda / m_{q^{*}}$ multiplied by the branching ratio of the $q^{*}$ into $\mathrm{q} \gamma$ and into $\mathrm{q} g$ respectively. These limits were obtained assuming up-type excited quarks. For down-type excited quarks the cross section limits are about 15\% higher in the studied mass region due to the lower expected production cross-section.

The lower limits at 95\% CL on the masses of pair-produced unstable heavy leptons are given in table 10. In the excited leptons case, limits are given for both $f=f^{\prime}$ and $f=-f^{\prime}$. In the case of the sequential leptons, decays into each of the leptonic families are considered.

Figure 7 shows the limit on the excited electron production for $f=f^{\prime}$ obtained by combining the result of the direct search (figure $\mathbb{G}(\mathrm{a})$ ) with the indirect result from the search for deviations in the $e^{+} e^{-} \rightarrow \gamma \gamma(\gamma)$ differential cross-section [11]. We can thus extend the limit to regions above the kinematic limit. 


\begin{tabular}{|c|c|c|c|c|}
\hline$\sqrt{s}$ & channel & $e$ & $\mu$ & $\tau$ \\
\hline \multirow{4}{*}{$\begin{array}{c}183 \\
\mathrm{Ge}\end{array}$} & $\overline{\ell^{*} \rightarrow \ell \gamma}$ & $(0.2 \pm 0.1)$ & $\overline{0(0.2 \pm 0.1)}$ & $\overline{1(0.9 \pm 0.2}$ \\
\hline & $\nu^{*} \rightarrow \nu \gamma$ & \multicolumn{3}{|c|}{$4(1.8 \pm 0.2)$} \\
\hline & $\ell^{*}, L^{ \pm}, E_{i}^{ \pm} \rightarrow \nu W$ & \multicolumn{3}{|c|}{$22(18 \pm 1)$} \\
\hline & $\nu^{*}, L^{0}, E_{i}^{0} \rightarrow \ell W$ & $5(2.6 \pm 0.5)$ & $5(2.9 \pm 0.5)$ & $17(13 \pm 1)$ \\
\hline \multirow{4}{*}{$\begin{array}{c}172 \\
\mathrm{GeV}\end{array}$} & $\ell^{*} \rightarrow \ell \gamma$ & $\overline{0(0.04 \pm 0.04)}$ & $\overline{0(0.04 \pm 0.04)}$ & $0(0.1 \pm 0.1)$ \\
\hline & $\nu^{*} \rightarrow \nu \gamma$ & \multicolumn{3}{|c|}{$2(0.8 \pm 0.1)$} \\
\hline & $\ell^{*}, L^{ \pm}, E_{i}^{ \pm} \rightarrow \nu W$ & \multicolumn{3}{|c|}{$5(4 \pm 0.4)$} \\
\hline & $\nu^{*}, L^{0}, E_{i}^{0} \rightarrow \ell W$ & $1(0.2 \pm 0.1)$ & $2(0.4 \pm 0.1)$ & $4(2.4 \pm 0.3$ \\
\hline \multirow{4}{*}{$\begin{array}{c}161 \\
\mathrm{GeV}\end{array}$} & $\ell^{*} \rightarrow \ell \gamma$ & $1(0.04 \pm 0.04)$ & $0(0.1 \pm 0.1)$ & $1(0.3 \pm 0.1$ \\
\hline & $\nu^{*} \rightarrow \nu \gamma$ & \multicolumn{3}{|c|}{$0(0.8 \pm 0.1)$} \\
\hline & $\ell^{*}, L^{ \pm}, E_{i}^{ \pm} \rightarrow \nu W$ & \multicolumn{3}{|c|}{$3(3.5 \pm 0.5)$} \\
\hline & $\nu^{*}, L^{0}, E_{i}^{0} \rightarrow \ell W$ & $0(0.5 \pm 0.2)$ & $0(0.4 \pm 0.1)$ & $(2.0+0.0$ \\
\hline
\end{tabular}

Table 8: Number of heavy lepton candidates for the different decay channels and the different centre-of-mass energies in the pair production modes. The numbers in brackets correspond to the simulated SM background expectations.

\section{Conclusions}

DELPHI data corresponding to integrated luminosities of $47.7 \mathrm{pb}^{-1}, 10 \mathrm{pb}^{-1}$ and 10 $\mathrm{pb}^{-1}$ at the centre-of-mass energies of $183 \mathrm{GeV}, 172 \mathrm{GeV}$ and $161 \mathrm{GeV}$ respectively have been analysed. A search for unstable heavy fermions decaying promptly through $\gamma, Z$, $W$ or gluon emission was performed. No significant signal was observed.

The search for pair production of heavy leptons resulted in the mass limits quoted in table 10. The search for single production of excited fermions gave the limits on the ratio $\lambda / m_{f}^{*}$ shown in Figures 0,0 , 6 and 0 . These results considerably extend the limits recently set from the runs of LEP at centre-of-mass energies of $161 \mathrm{GeV}$ and $172 \mathrm{GeV}$ or previously at LEP1 and HERA [2,0,8,[1]. 


\begin{tabular}{|l|c|c|c|}
\hline channel & $e$ & $\mu$ & $\tau$ \\
\hline \hline$\ell^{*} \rightarrow \ell \gamma$ & 33 & 59 & 33 \\
\hline$\ell^{*} \rightarrow \nu W$ & 21 & 43 & 32 \\
\hline$\ell^{*} \rightarrow \ell Z$ & 26 & 53 & 23 \\
\hline \hline$\nu^{*} \rightarrow \nu \gamma$ & \multicolumn{3}{|c|}{42} \\
\hline$\nu^{*} \rightarrow \ell W$ & 44 & 48 & 17 \\
\hline$\nu^{*} \rightarrow \nu Z$ & \multicolumn{3}{|c|}{21} \\
\hline \hline$q^{*} \rightarrow q \gamma$ & \multicolumn{3}{|c|}{31} \\
\hline$q^{*} \rightarrow q g$ & \multicolumn{3}{|c|}{19} \\
\hline
\end{tabular}

\begin{tabular}{|l|c|c|c|}
\hline channel & $e$ & $\mu$ & $\tau$ \\
\hline \hline$\ell^{*} \rightarrow \ell \gamma$ & 28 & 37 & 26 \\
\hline$\nu^{*} \rightarrow \nu \gamma$ & \multicolumn{3}{|c|}{46} \\
\hline \hline$\ell^{*}, L^{ \pm}, E_{i}^{ \pm} \rightarrow \nu W$ & \multicolumn{3}{|c|}{14} \\
\hline$\nu^{*}, L^{0}, E_{i}^{0} \rightarrow \ell W$ & 27 & 37 & 20 \\
\hline
\end{tabular}

Table 9: Efficiencies (in percentage) for the single (upper) and pair (lower) production modes at a centre-of-mass energy of $183 \mathrm{GeV}$. The values were obtained with excited lepton masses of $170 \mathrm{GeV} / c^{2}$ and $80 \mathrm{GeV} / c^{2}$ for single and pair production respectively.

\section{Acknowledgements}

We would like to thank Jorge Romão for the very useful discussions.

We are greatly indebted to our technical collaborators, to the members of the CERNSL Division for the excellent performance of the LEP collider, and to the funding agencies for their support in building and operating the DELPHI detector.

We acknowledge in particular the support of Austrian Federal Ministry of Science and Traffics, GZ 616.364/2-III/2a/98, FNRS-FWO, Belgium, FINEP, CNPq, CAPES, FUJB and FAPERJ, Brazil, Czech Ministry of Industry and Trade, GA CR 202/96/0450 and GA AVCR A1010521, Danish Natural Research Council,

Commission of the European Communities (DG XII), Direction des Sciences de la Matière, CEA, France, Bundesministerium für Bildung, Wissenschaft, Forschung und Technologie, Germany, General Secretariat for Research and Technology, Greece,

National Science Foundation (NWO) and Foundation for Research on Matter (FOM), The Netherlands,

Norwegian Research Council,

State Committee for Scientific Research, Poland, 2P03B06015, 2P03B03311 and SPUB/P03/178/98,

JNICT-Junta Nacional de Investigação Científica e Tecnológica, Portugal,

Vedecka grantova agentura MS SR, Slovakia, Nr. 95/5195/134,

Ministry of Science and Technology of the Republic of Slovenia,

CICYT, Spain, AEN96-1661 and AEN96-1681,

The Swedish Natural Science Research Council,

Particle Physics and Astronomy Research Council, UK, 


\begin{tabular}{|c|c|c|c|}
\hline$L^{ \pm}$ & $L^{0} \rightarrow e W$ & $L^{0} \rightarrow \mu W$ & $L^{0} \rightarrow \tau W$ \\
\hline \hline 78.3 & 76.5 & 79.5 & 60.5 \\
\hline
\end{tabular}

\begin{tabular}{|l||c|c|c|c|}
\hline & $E_{i}$ & $N_{e}$ & $N_{\mu}$ & $N_{\tau}$ \\
\hline \hline vector & 81.3 & 87.3 & 88.0 & 81.0 \\
\hline mirror & 78.3 & 76.5 & 79.5 & 60.5 \\
\hline
\end{tabular}

\begin{tabular}{|c||c|c|c||c||c|c|c|}
\hline & $e^{*}$ & $\mu^{*}$ & $\tau^{*}$ \\
\hline \hline$f=f^{\prime}$ & 90.7 & 90.7 & 89.7 \\
\hline \hline$f=f^{\prime}$ & $\nu_{e}^{*}$ & $\nu_{\mu}^{*}$ & $\nu_{\tau}^{*}$ \\
\hline$f=-f^{\prime}$ & 81.3 & 81.3 & 81.3 & 88.0 & 81.0 \\
\hline$f=-f^{\prime}$ & 90.0 & 90.0 & 90.0 \\
\hline
\end{tabular}

Table 10: Lower limits (in $\mathrm{GeV} / c^{2}$ ) at $95 \% \mathrm{CL}$ on the unstable heavy lepton masses from the pair production modes. Starting from the top, the three tables correspond to sequential, non-canonical and excited leptons respectively.

Department of Energy, USA, DE-FG02-94ER40817. 


\section{References}

[1] "Searches for new Physics" G.F.Giudice et al., Physics at LEP2, vol. 1, eds. G.Altarelli et al., CERN Yellow Book CERN-96-01 (1996) 463.

[2] DELPHI Coll., P. Abreu et al., Phys. Lett. B393 (1997) 245.

[3] G. Bhattacharyya, Nucl. Phys. B468 (1996) 59. (CERN-TH-95-306)

[4] A. Djouadi, Zeit. Phys. C63 (1994) 317.

[5] "New Particles and interactions" A. Djouadi, J. Ng and T.G. Rizzo, hep-ph/9504210, (1995), SLAC-PUB-95-6772, to appear as a chapter in "Electroweak symmetry breaking and beyond the Standard Model", edited by T. Barlow, S. Dawson, H.E. Haber and S. Siegrist, World Scientific.

[6] K. Hagiwara, S. Komamiya and D. Zeppenfeld, Z. Phys. C29 (1985) 115;

F. Boudjema, A. Djouadi and J.L. Kneur, Z. Phys. C57 (1993) 425.

[7] DELPHI Coll., P. Abreu et al., Zeit. Phys. C 74 (1997) 57;

DELPHI Coll., P. Abreu et al., Zeit. Phys. C 74 (1997) 577;

DELPHI Coll., P. Abreu et al., Zeit. Phys. C53 (1992) 41;

DELPHI Coll., P. Abreu et al., Phys. Lett. B274 (1992) 230.

[8] ALEPH Coll., D. Buskulic et al., Phys. Lett. B385 (1996) 445;

ALEPH Coll., D. Buskulic et al., Phys. Lett. B384 (1996) 439;

ALEPH Coll., D. Decamp et al., Phys. Lett. B250 (1990) 172;

ALEPH Coll., D. Decamp et al., Phys. Lett. B236 (1990) 501;

ALEPH Coll., D. Decamp et al., Phys. Lett. B236 (1990) 511;

H1 Coll., I. Abt et al., Nucl. Phys. B396 (1993) 3;

L3 Coll., M. Acciarri et al., Phys. Lett. B412 (1997) 189;

L3 Coll., M. Acciarri et al., Phys. Lett. B401 (1997) 139;

L3 Coll., M. Acciarri et al., Phys. Lett. B377 (1996) 304;

L3 Coll., M. Acciarri et al., Phys. Lett. B370 (1996) 211;

L3 Coll., O. Adriani et al., Phys. Lett. B295 (1992) 371;

L3 Coll., B. Adeva et al., Phys. Lett. B251 (1990) 321;

L3 Coll., B. Adeva et al., Phys. Lett. B247 (1990) 177;

L3 Coll., B. Adeva et al., Phys. Lett. B250 (1990) 205;

L3 Coll., B. Adeva et al., Phys. Lett. B252 (1990) 525;

OPAL Coll., K. Ackerstaff et al., Eur. Phys. J. C1 (1998) 45;

OPAL Coll., K. Ackerstaff et al., Phys. Lett. B393 (1997) 217;

OPAL Coll., K. Ackerstaff et al., Phys. Lett. B391 (1997) 197;

OPAL Coll., G. Alexander et al., Phys. Lett. B386 (1996) 463;

OPAL Coll., G. Alexander et al., Phys. Lett. B385 (1996) 433;

OPAL Coll., G. Alexander et al., Z.Phys. C52 (1991) 175;

OPAL Coll., M.Z. Akrawy et al., Phys. Lett. B257 (1991) 531;

OPAL Coll., M.Z. Akrawy et al., Phys. Lett. B247 (1990) 448;

OPAL Coll., M.Z. Akrawy et al., Phys. Lett. B244 (1990) 135;

OPAL Coll., M.Z. Akrawy et al., Phys. Lett. B240 (1990) 250;

ZEUS Coll., M. Derrick et al., Z. Phys. C65 (1994) 627.

[9] "Exotic Fermions", D.London, Precision Tests of the Standard Model, 1993, ed. P.Langacker, World Scientific.

[10] DELPHI Coll., P. Abreu et al., Phys. Lett. B380 (1996) 480.

[11] DELPHI Coll., P. Abreu et al., Eur. Phys. J. C1 (1998) 1;

DELPHI Coll., P. Abreu et al., Phys. Lett. B433 (1998) 429;

DELPHI Coll., P. Abreu et al., Phys. Lett. B380 (1996) 471; 
[12] DELPHI Coll., P. Aarnio et al., Nucl. Instr. Methods A303 (1991) 233;

DELPHI Coll., P. Abreu et al., Nucl. Instr. Methods A378 (1996) 57.

[13] F.A. Berends, W. Hollik and R. Kleiss, Nucl. Phys. B304 (1988) 712.

[14] T. Sjöstrand, Comp. Phys. Comm. 82 (1994) 74.

[15] S. Jadach, B.F.L. Ward and Z. Wa̧s, Comp. Phys. Comm 66 (1991) 276.

[16] F.A. Berends, R. Pittau and R. Kleiss, Comp. Phys. Comm 85 (1995) 437.

[17] S. Nova, A. Olchevski and T. Todorov, "TWOGAM, CERN report 96-01, vol.2 p. 224 (1996).

[18] F.A. Berends, P.H. Daverveldt and R. Kleiss, Comp. Phys. Comm. 40 (1986) 271.

[19] D. Karlen, Nucl. Phys. B289 (1987) 23.

[20] F. Berends and R. Kleiss, Nucl. Phys. B186 (1981) 22.

[21] S. Catani et al., Phys. Lett. B269 (1991) 432.

[22] DELPHI Coll., P. Abreu et al., Eur. Phys. J. C2 (1998) 581.

[23] A.L. Read, "Optimal statistical analysis of search results based on the likelihood ratio and its application to the search for the MSM Higgs boson at $\sqrt{s}=161$ and 172 GeV", DELPHI note 97-158 PHYS 737. 

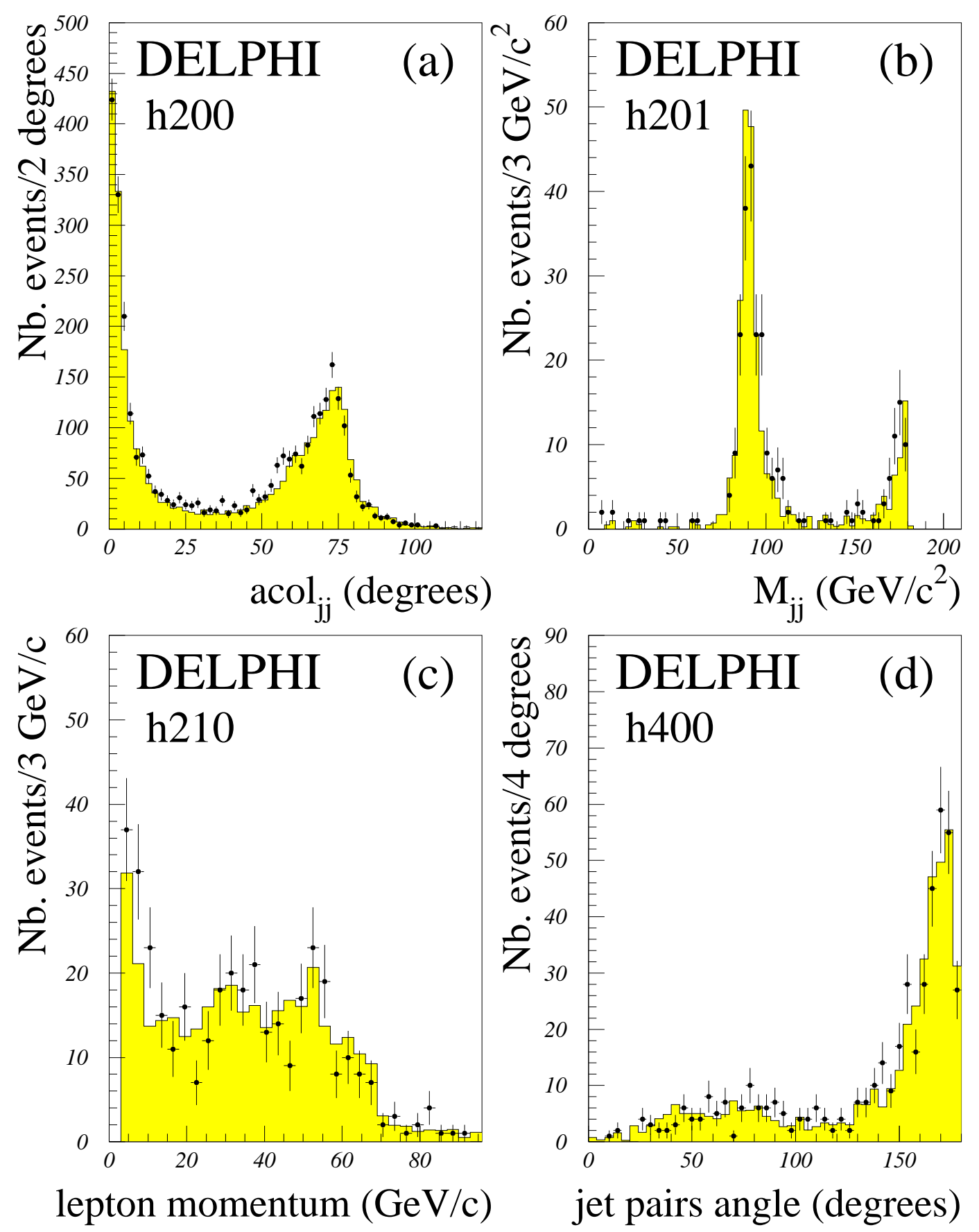

Figure 1: Acolinearity (a) and invariant mass (b) of the two jets in the h200 and h201 topologies respectively, momentum of the lepton in the h210 topology (c), angle between the two jet pairs in the h400 topology (d) at $183 \mathrm{GeV}$. The dots show the data and the shaded histograms show the SM simulation. 



Figure 2: Jet-jet (a) and jet-photon (b) invariant mass in the h300 and h201 topologies respectively, lepton recoil mass (c) and jet-jet-lepton invariant mass (d) in the h210 topology at $183 \mathrm{GeV}$. The dots show the data and the shaded histograms show the SM simulation. 

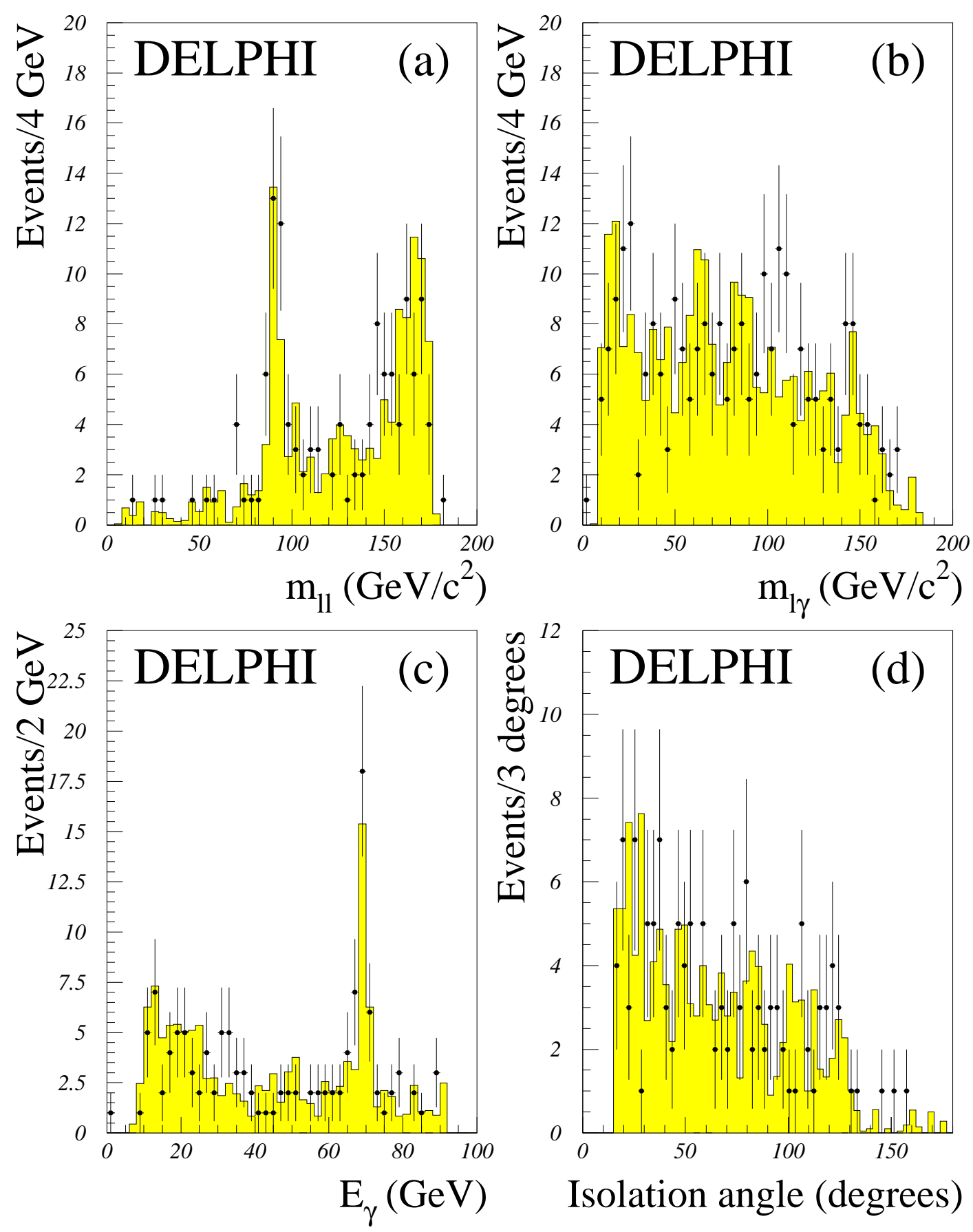

Figure 3: Invariant mass of the two leptons (a), invariant mass of lepton-photon pairs (b), and energy (c) and isolation angle (d) of the photon, for the $\ell 201$ topology at $183 \mathrm{GeV}$. The dots show the data and the shaded histograms show the SM simulation. 

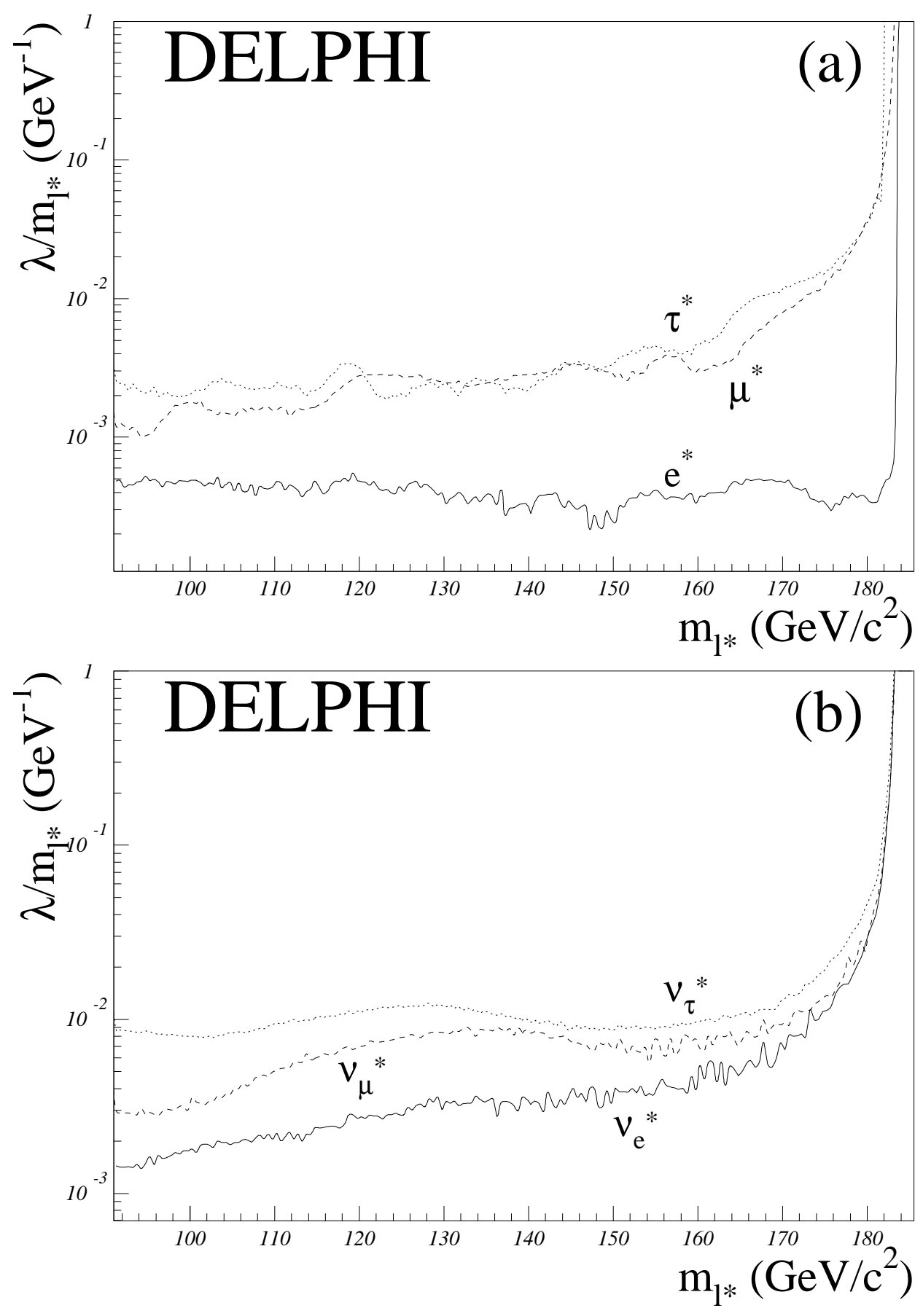

Figure 4: Results on single production of excited charged (a) and neutral (b) leptons assuming $f=+f^{\prime}$. The lines show the upper limits at $95 \% \mathrm{CL}$ on the ratio $\lambda / m_{\ell^{*}}$ between the coupling of the excited lepton and its mass as a function the mass. 

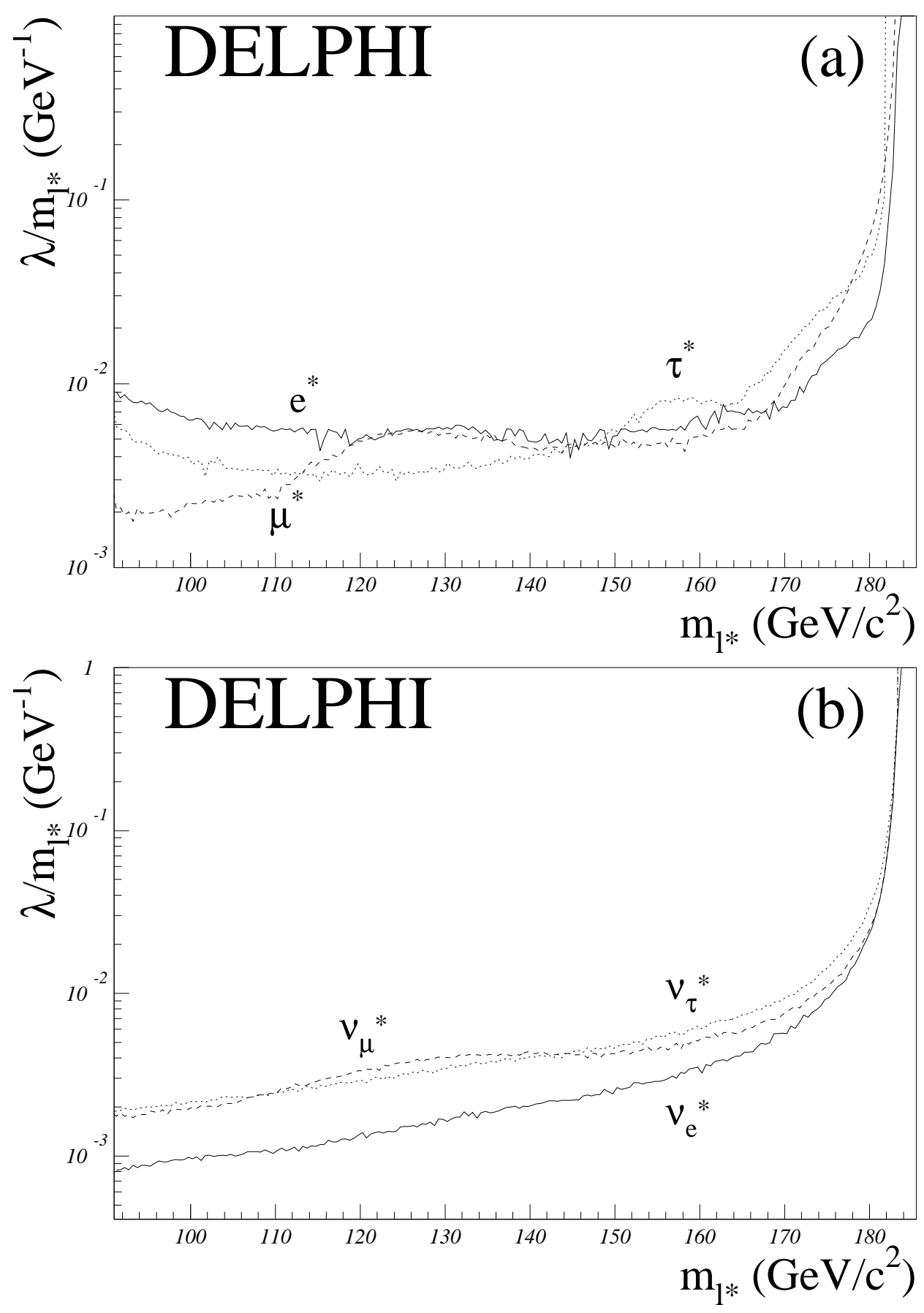

Figure 5: As figure 4, but for $f=-f^{\prime}$. 

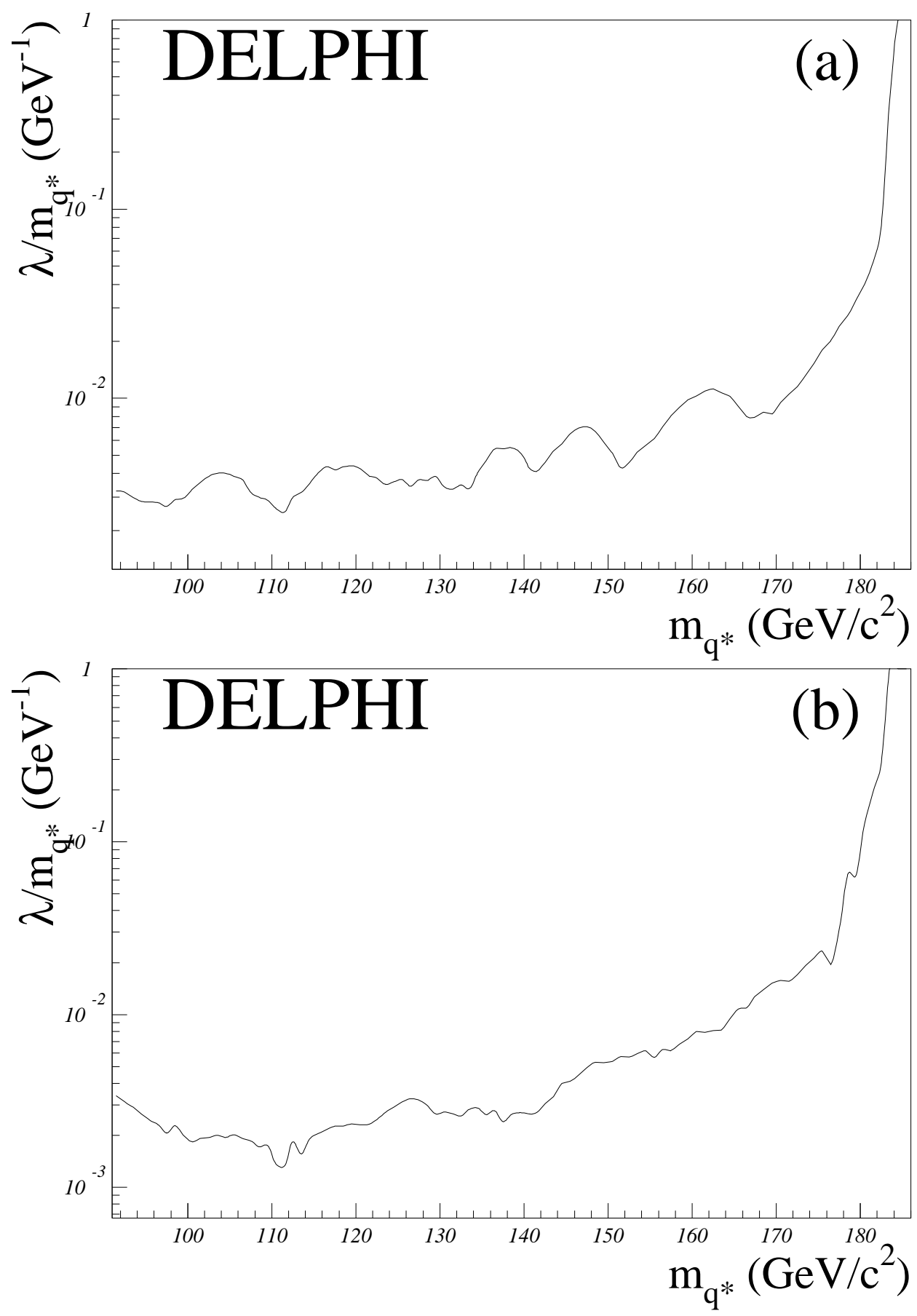

Figure 6: Results on single production of excited quarks assuming a branching ratio of $100 \%$ for the photon (a) or the gluon (b) decay modes. The lines show the upper limits at $95 \%$ CL on the ratio $\lambda / m_{q^{*}}$ between the coupling of the excited quark and its mass as a function of the mass. 


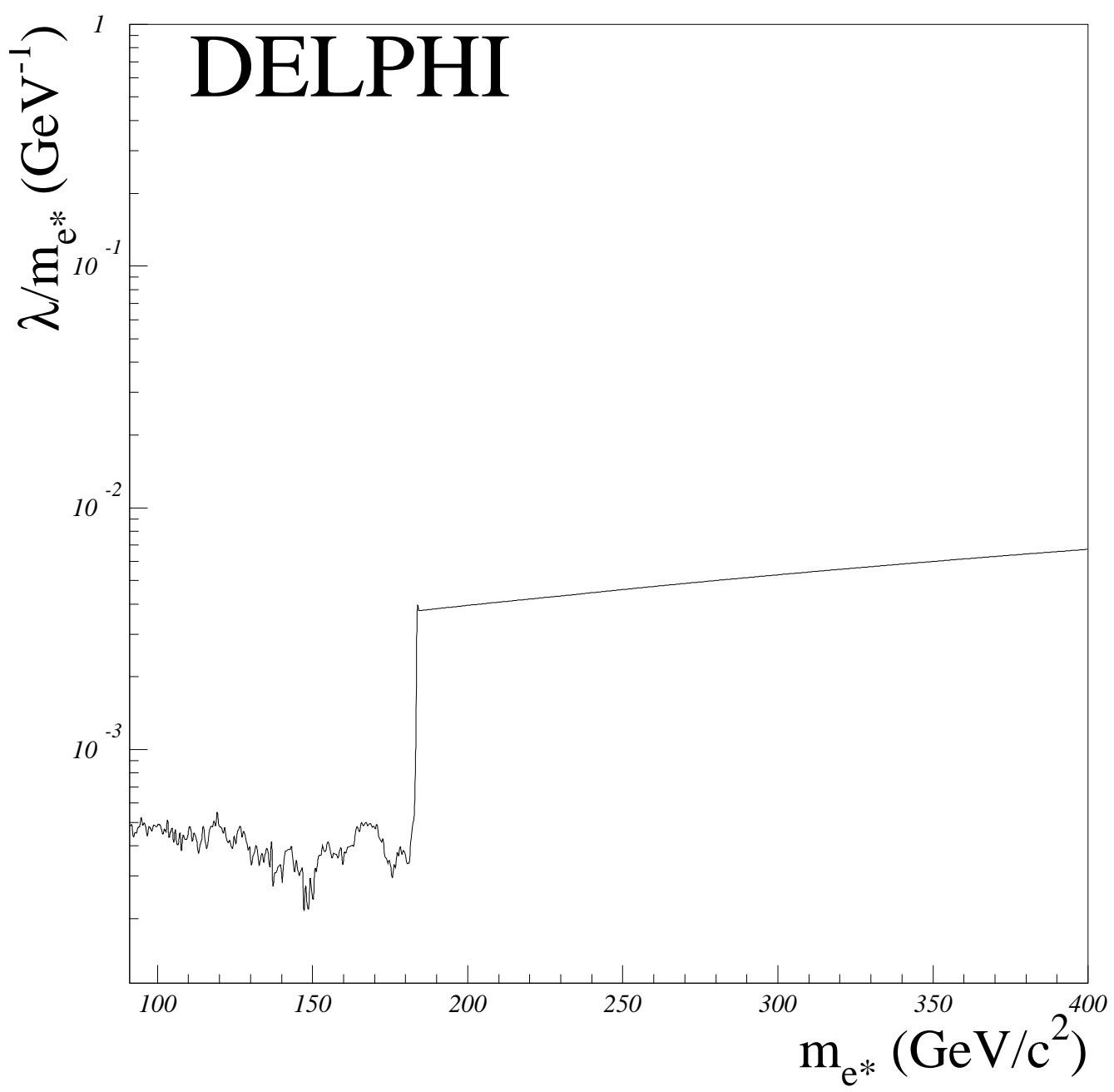

Figure 7: Combined excited electron limits for $f=f^{\prime}$ from direct and indirect searches. The line shows the upper limits at $95 \%$ CL on the ratio $\lambda / m_{e^{*}}$ between the coupling of the excited electron and its mass as a function of the mass. Up to the kinematic limit the result is dominated by the single production direct search. Above this value the limit it is the one coming from the indirect search using $e^{+} e^{-} \rightarrow \gamma \gamma$. 